\title{
INFRARED PROPERTIES OF CLOSE PAIRS OF GALAXIES
}

\author{
Margaret J. Geller and Scott J. Kenyon \\ Smithsonian Astrophysical Observatory, 60 Garden Street, Cambridge, MA 02138; mgeller@cfa.harvard.edu, skenyon@cfa.harvard.edu
}

Elizabeth J. Barton

Department of Physics and Astronomy, University of California at Irvine, 4154 Frederick Reines Hall, Irvine, CA 92697; ebarton@uci.edu

ThOMAS H. JaRRETT

Infrared Processing and Analysis Center, Spitzer Science Center, Jet Propulsion Laboratory, California Institute of Technology, Pasadena, CA 91125; jarrett@ipac.caltech.edu

AND

LisA J. KeWLEY

Institute for Astronomy, University of Hawaii, 2680 Woodlawn Drive, Manoa, HI 96822; kewley@ifa.hawaii.edu Received 2006 April 13; accepted 2006 August 1

\begin{abstract}
We discuss spectroscopy and IR photometry for a complete sample of $\sim 800$ galaxies in close pairs objectively selected from the second Center for Astrophysics redshift survey. We use the Two Micron All Sky Survey to compare near-IR color-color diagrams for our sample with the Nearby Field Galaxy Sample and with a set of IRAS fluxlimited pairs from Surace and coworkers. We construct a basic statistical model to explore the physical sources of the substantial differences among these samples. The model explains the spread of near-IR colors and is consistent with a picture in which central star formation is triggered by the galaxy-galaxy interaction before a merger occurs. For 160 galaxies we report new, deep $J H K$ photometry within our spectroscopic aperture, and we use the combined spectroscopic and photometric data to explore the physical conditions in the central bursts. We find a set of objects with $H-K \geq 0.45$ and with a large $F_{\mathrm{FIR}} / F_{H}$. We interpret the very red $H-K$ colors as evidence for $600-1000 \mathrm{~K}$ dust within compact star-forming regions, perhaps similar to super star clusters identified in individual well-studied interacting galaxies. The galaxies in our sample are candidate "hidden" bursts or, possibly, "hidden" active galactic nuclei. Over the entire pair sample, both spectroscopic and photometric data show that the specific star formation rate decreases with the projected separation of the pair. The data suggest that the near-IR color-color diagram is also a function of the projected separation; all of the objects with central near-IR colors indicative of bursts of star formation lie at small projected separation.
\end{abstract}

Key words: galaxies: interactions — galaxies: photometry — galaxies: starburst

Online material: machine-readable tables

\section{INTRODUCTION}

Larson \& Tinsley (1978) first recognized the close connection between galaxy-galaxy interactions and star formation. They suggested that this relationship is a fundamental ingredient of galaxy formation and evolution. Studies of individual interacting systems and of statistical samples of close pairs in the local universe have confirmed and refined the initial results (e.g., Kennicutt \& Keel 1984; Kennicutt et al. 1987; Jones \& Stein 1989; Sekiguchi \& Wolstencroft 1992; Keel 1993, 1996; Liu \& Kennicutt 1995a, 1995b; Donzelli \& Pastoriza 1997; Barton et al. 2000, hereafter BGK00; Barton Gillespie et al. 2003, hereafter BGK03; Lambas et al. 2003; Allam et al. 2004; Alonso et al. 2004; Nikolic et al. 2004). Examinations of the universe at high redshift underscore the importance of interactions in molding the evolution of galaxies (e.g., Patton et al. 2002; Conselice et al. 2004; Lin et al. 2004; Papovich et al. 2005; Bell et al. 2006).

The connection between interacting pairs of galaxies and star formation is now well established observationally, and there is a promising correspondence between the predictions of simulations and the properties of well-defined samples of close pairs. The data are consistent with a burst of star formation triggered by a close galaxy-galaxy interaction; the burst continues and ages as the galaxies move apart (Mihos et al. 1991; Mihos \& Hernquist 1996; BGK00; BGK03; Lambas et al. 2003; Nikolic et al. 2004). The primary feature of the data is an anticorrelation between the projected separation of a pair on the sky and measures of the normalized star formation rate. This anticorrelation suggests a dependence of the burst strength and probably burst age on the separation of the galaxy pair (BGK03).

Here we focus on near-IR (NIR) observations of close pairs of galaxies in the full BGK00 sample with attention to the distinctive features of IR emission possibly associated with the interaction. The literature contains detailed NIR observations of famous individual interacting galaxy pairs (e.g., López-Sánchez et al. 2004) and a few optical-IR imaging surveys of small, objectively selected samples of close pairs. Bushouse \& Werner (1990) imaged a sample of 22 interacting galaxies. In their sample, the nuclei are redder than the outer regions by $\sim 0.4$ and 0.9 mag in $J-K$ and $H-K$, respectively. They attribute the color gradient mostly to an increase in the amount of dust in the nuclear regions and argue that the IR colors are unaffected by dust emission. Cutri \& McAlary (1985) carried out small-aperture photometry of a larger sample of pairs. They concluded that a larger fraction of interacting than noninteracting galaxies have $J-K$ and $H-K$ colors outside the normal range. Giuricin et al. (1993) used a compendium of data from the literature to investigate 
the effect of interactions on the NIR properties of spiral galaxies. In contrast with Bushouse \& Werner (1990), they concluded that apparently interacting galaxies have $H-K$ and $K-L$ excesses indicative of thermal emission from hot dust probably related to star formation induced by the interaction. We use our spectroscopy and matched small-aperture photometry along with Two Micron All Sky Survey (2MASS) photometry to revisit these issues in a much larger data set of nearly 800 galaxies in close pairs and $n$-tuples.

In the far-IR (FIR), IRAS observations still yield the largest data sets for consideration of the properties of galaxy-galaxy interactions as a function of observable descriptors of the pair (see, e.g., Goto 2005). Telesco et al. (1988) compiled IRAS observations of pairs selected from the catalog of Arp \& Madore (1987) and resolved by IRAS. In their set of 93 pairs of comparable luminosity, they found that pairs with the highest FIR color temperature have the smallest projected separation on the sky. They concluded that interactions measurably enhance the intensity or efficiency of star formation. Bushouse et al. (1988) reached similar conclusions based on IRAS observations of a sample of pairs selected on the basis of optical morphology.

Recently, Surace et al. (2004, hereafter SSM04) used High Resolution Echelle Spectrometer image reconstruction to resolve the IRAS emission at 12, 25, 60, and $100 \mu \mathrm{m}$ for 106 interacting galaxy systems in which the galaxies are separated by less than three average galaxy diameters. The sample has a flux limit at $60 \mu \mathrm{m}$ of $5.24 \mathrm{Jy}$. We use their sample to provide a context for discussion of the generally unresolved IRAS detections in our sample. Neither the SSM04 nor the BGK00 samples contain any ultraluminous IR galaxies (ULIRGs).

The complexity of the underlying sources of emission in the NIR has probably been a disincentive to investigating pairs in this spectral range. With current data it remains difficult to disentangle the effects of reddening and thermal dust emission at several temperatures. Photometry at $L$ together with spatially resolved photometry of large samples at longer wavelengths is necessary for a clearer understanding of these issues. Currently available data are, however, adequate to demonstrate the promise of the IR for probing the galaxy-galaxy merger process and the links between galaxy-galaxy interactions and star formation.

Here we compile the largest sample to date of the NIR colors of galaxy pairs. The data we consider include $2 \mathrm{MASS} J H K_{s}$ photometry for 791 galaxies in the BGK00 sample and our own deeper $J H K$ small-aperture photometry for a subsample of 160 BGK00 galaxies. We also use IRAS detections and upper limits for the entire BGK00 sample as a marker of the relationship between galaxy-galaxy interactions, star formation, and thermal emission from hot dust in these systems. We use our optical spectra and IR colors measured through the same aperture to investigate basic properties of the central regions of these galaxies.

Section 2 is a description of the IR photometry and a review of the optical photometry and spectroscopy. The data include resolved 2MASS photometry for 44 systems unresolved in the public catalogs and for 24 galaxies not detected or confused with nearby stars or galaxies. In $\S 3$ we investigate the IR properties of the sample. We discuss the NIR color-color diagram and investigate the relationships among the NIR and FIR colors, the Balmer decrement, and the normalized star formation rate. In $\S 4$ we show that the star formation rate as indicated by the normalized FIR flux increases as the projected pair separation decreases as expected if the interaction triggers star formation. There is also a suggestive increase in the spread of NIR color as the projected separation of the pair decreases. This increased spread is evidence for triggered central bursts that may be either very blue or very heavily obscured and reddened. We conclude in $\oint 5$.

\section{OBSERVATIONS AND DATA REDUCTION}

\subsection{The Sample of Pairs}

BGK00 identified 358 close pairs and $n$-tuples in the original magnitude-limited second Center for Astrophysics (CfA2) redshift survey with $m_{\mathrm{Zw}} \leq 15.5$. The original CfA2North survey covers the declination range $8^{\circ} .5<\delta_{\mathrm{B} 1950.0}<44^{\circ} .5$ and right ascension range $8^{\mathrm{h}}<\alpha_{\mathrm{B} 1950.0}<17^{\mathrm{h}}$ and includes 6500 galaxies (Geller \& Huchra 1989; Huchra et al. 1990, 1995). The original CfA2South survey covers the region $\delta=-2.5<\delta_{\mathrm{B} 1950.0}<$ $48^{\circ}$ and $\alpha=20^{\mathrm{h}}<\alpha_{\mathrm{B} 1950.0}<4^{\mathrm{h}}$ and includes 4283 galaxies (Giovanelli \& Haynes 1985, 1989, 1993; Giovanelli et al. 1986; Haynes et al. 1988; Wegner et al. 1993; Huchra et al. 1999; see also Falco et al. [1999] for updated data for CfA2North and CfA2South).

The pairs were originally selected with line-of-sight velocity separations $\Delta V<1000 \mathrm{~km} \mathrm{~s}^{-1}$, projected separations $\Delta D<$ $50 h^{-1} \mathrm{kpc}$, and $c z>2300 \mathrm{~km} \mathrm{~s}^{-1}$. In this pair sample, $n$-tuples result from pairs linked by common members as "friends-offriends." The $2300 \mathrm{~km} \mathrm{~s}^{-1}$ limit excludes the Virgo Cluster and limits the angular sizes of the galaxies relative to the slit aperture we use for spectroscopy $(\S 2.5)$. We use a Hubble constant $100 h \mathrm{~km} \mathrm{~s}^{-1} \mathrm{Mpc}^{-1}$ unless otherwise specified.

Ninety percent of the pairs have $\Delta V<500 \mathrm{~km} \mathrm{~s}^{-1}$, comparable to the typical pairwise velocity dispersion in the redshift survey (Marzke et al. 1995). We repeated all of the analysis below for pair samples restricted to $\Delta V<500 \mathrm{~km} \mathrm{~s}^{-1}(89 \%$ of the original sample) and $\Delta V<400 \mathrm{~km} \mathrm{~s}^{-1}$ (84\% of the original sample); there are no significant differences in any results for any sample.

The pairs with $\Delta V>500 \mathrm{~km} \mathrm{~s}^{-1}$ appropriately lie within rich clusters in which the local velocity dispersion exceeds the mean and the local density contrast substantially exceeds the mean; we include these pairs for completeness and for consistency with our previous analyses of this catalog. We use the BGK00 technique based on an estimate of the galaxy overdensity in a $5 h^{-1} \mathrm{Mpc}$ sphere around each pair in the sample to compute the interloper fraction for the entire pairs sample, $\lesssim 20 \%$. By restricting the pairs sample to regions of low density contrast, we also repeated our entire analysis for samples in which the estimated interloper fraction is $\lesssim 10 \%$. These restricted pair samples yield the same results we obtain for the full sample and provide reassurance that interlopers are not responsible for salient differences between the NIR characteristics of pairs and the general galaxy population.

The pairs in the full sample we use were selected without explicit bias in morphology or environment. Updated coordinates and redshifts modify the sample slightly from the original one discussed in BGK00; the sample we analyze here contains 791 rather than the 786 galaxies in BGK00. These galaxies lie in 306 pairs, 37 triples, 8 quadruples, and 7 quintuples. From here on, for simplicity, we refer to all of these systems as pairs.

\subsection{MASS Photometry}

2MASS is an all sky survey with uniform, complete photometry (Nikolaev et al. 2000; Skrutskie et al. 2006) in three IR bands, $J, H$, and $K_{s}$. Here $K_{s}$ is a modified version of the $K$ filter designed specifically to reduce thermal background. For most of our analysis, we use the $20 \mathrm{mag} \operatorname{arcsec}^{-2}$ isophotal elliptical aperture photometry from the final extended source catalog 
TABLE 1

2MASS INFRARED PHOTOMETRY

\begin{tabular}{|c|c|c|c|c|c|c|c|c|}
\hline \multirow{2}{*}{$\begin{array}{c}\mathrm{J} 2000.0 \\
\text { Designation } \\
\text { (1) }\end{array}$} & \multirow[b]{2}{*}{$\begin{array}{c}\text { ReDSHIFT } \\
\text { (2) }\end{array}$} & \multicolumn{3}{|c|}{$7^{\prime \prime}$ Aperture } & \multicolumn{3}{|c|}{ IsOPHOTAL APERTURe } & \multirow[b]{2}{*}{$\begin{array}{c}\text { SPLIT }^{\mathrm{a}} \\
(9)\end{array}$} \\
\hline & & $\begin{array}{c}J \\
(3)\end{array}$ & $\begin{array}{c}H \\
(4)\end{array}$ & $\begin{array}{c}K \\
(5)\end{array}$ & $\begin{array}{c}J \\
(6)\end{array}$ & $\begin{array}{c}H \\
(7)\end{array}$ & $\begin{array}{c}K \\
(8)\end{array}$ & \\
\hline $08042396+2930516 \ldots \ldots \ldots \ldots \ldots$ & 5447 & 13.87 & 13.22 & 12.90 & 13.51 & 12.91 & 12.60 & \\
\hline $08042496+2930236 \ldots \ldots \ldots \ldots \ldots$ & 5298 & 12.11 & 11.40 & 11.15 & 11.80 & 11.12 & 10.87 & \\
\hline $08065208+1844155 \ldots \ldots \ldots \ldots \ldots$ & 4557 & 12.03 & 11.32 & 11.05 & 11.72 & 11.02 & 10.74 & \\
\hline $08070665+1845506 \ldots \ldots \ldots \ldots \ldots$ & 4661 & 13.98 & 13.46 & 13.18 & 13.62 & 13.02 & 12.76 & \\
\hline $08100603+2455194 \ldots \ldots \ldots \ldots \ldots$ & 4128 & 12.70 & 11.97 & 11.71 & 12.19 & 11.47 & 11.24 & \\
\hline 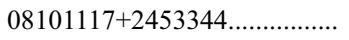 & 4089 & 14.36 & 13.76 & 13.51 & 13.72 & 13.11 & 12.80 & \\
\hline $08111348+2512249 \ldots \ldots \ldots \ldots \ldots$ & 3995 & 12.82 & 12.10 & 11.80 & 11.35 & 10.66 & 10.40 & \\
\hline $08111591+2510459 \ldots \ldots \ldots \ldots \ldots$ & 4039 & 13.15 & 12.53 & 12.27 & 12.79 & 12.18 & 11.88 & \\
\hline $08112548+0853382 \ldots \ldots \ldots \ldots \ldots$ & 5763 & 14.09 & 13.45 & 13.15 & 14.31 & 13.66 & 13.34 & + \\
\hline $08112703+0856280 \ldots \ldots \ldots \ldots \ldots$ & 5704 & 14.00 & 13.32 & 13.05 & 13.86 & 13.19 & 12.97 & \\
\hline
\end{tabular}

Notes.-Table 1 is published in its entirety in the electronic edition of the Astronomical Journal. A portion is shown here for guidance regarding its form and content.

a Asterisks indicate previously unresolved objects. Twenty-four pair galaxies are either undetected or confused with nearby stars or nearby nonpair galaxies. T. H. J. also reanalyzed these pairs, which are indicated by plus signs.

(Jarrett et al. 2000). We compare slit $J, H$, and $K$ magnitudes $(\S 2.3)$ with the 2MASS $7^{\prime \prime}$ aperture magnitudes.

We downloaded $J H K_{s}$ from the 2MASS extended source catalog using the Infrared Science Archive (IRSA) Web interface at IPAC. ${ }^{1}$ All 791 galaxies in the complete pairs sample are detected.

Among the BGK00 pairs, 44 systems (12\%) are unresolved in 2MASS. The median recessional velocity, $c z$, of the BGK00 sample is only $5852 \mathrm{~km} \mathrm{~s}^{-1}$. The unresolved pairs are a potentially serious problem in trying to use the 2MASS catalog for pair selection even at this depth and certainly as a basis for a deeper sample. One of us (T. H. J.) reanalyzed the 2MASS data to obtain magnitudes for the individual galaxies in the unresolved BGK00 pairs. For each galaxy, Table 1 lists the J2000.0 coordinate (col. [1]); the heliocentric radial velocity, $c z$ (col. [2]); the $J, H$, and $K_{s} 7^{\prime \prime}$ aperture magnitudes (cols. [3], [4], and [5], respectively); and the $J, H$, and $K_{s} 20 \mathrm{mag} \operatorname{arcsec}^{-2}$ isophotal elliptical magnitudes (cols. [6], [7], and [8], respectively). We indicate the previously unresolved objects with asterisks in column (9). Twenty-four pair galaxies are either undetected or confused with nearby stars or nearby nonpair galaxies. T. H. J. also reanalyzed these pairs, which are indicated by plus signs in column (9).

For 2MASS galaxies with $J \approx 11-13$ the photometric errors are $\sigma_{J} \approx 0.02-0.04, \sigma_{H} \approx 0.03-0.05$, and $\sigma_{K_{s}} \approx 0.04-0.06$ for the isophotal magnitudes and $\sigma_{J} \approx 0.01-0.03, \sigma_{H} \approx 0.02-0.04$, and $\sigma_{K_{s}} \approx 0.03-0.05$ for the $7^{\prime \prime}$ aperture magnitudes. To estimate the uncertainty in the colors, we compute the average color differences

$$
a_{J-H}=\left\langle\left|(J-H)_{7}-(J-H)_{\text {iso }}\right|\right\rangle
$$

and

$$
a_{H-K}=\left\langle\left|(H-K)_{7}-(H-K)_{\text {iso }}\right|\right\rangle .
$$

The subscript "7" refers to the 7" aperture colors, and the subscript "iso" refers to the isophotal colors. For galaxies with

\footnotetext{
${ }^{1}$ See http://irsa.ipac.caltech.edu.
}

$J \approx 11-13, a_{J-H}=0.026$ and $a_{H-K}=0.034$. Thus, the uncertainty in the typical $2 \mathrm{MASS}$ color is $\sim 0.03 \mathrm{mag}$. This approach to the calculation of the error in the colors follows a procedure recommended by Press et al. (1992).

To compare the NIR properties of the BGK00 sample with the general galaxy population, we use the Nearby Field Galaxy Sample (NFGS; Jansen et al. 2000a, 2000b). The NFGS was also selected from the Zwicky catalog, but the selection reproduces the galaxy luminosity function. Because it is drawn from a magnitude-limited survey, the BGK00 sample is biased against the lowest luminosity galaxies sampled by the NFGS. We extract 2MASS magnitudes for the NFGS galaxies, and we compare the NIR colors of the BGK00 pairs with the appropriate sample of representative NFGS galaxies. We restrict this comparison to the $H$-band luminosity range $\log \left(L_{H}\right)=8.75-11.7 L_{\odot}\left(H_{0}=\right.$ $73 \mathrm{~km} \mathrm{~s}^{-1} \mathrm{Mpc}^{-1}$ ) covered by both samples. There are $181 \mathrm{gal}-$ axies in the NFGS comparison sample.

\subsection{Near-Infrared Photometry}

We acquired $J H K$ images of 160 pair galaxies in the sample of BGK03 along with standard stars. These pairs are a random selection of the BGK00 pairs. We used several NIR cameras at the Fred L. Whipple Observatory $1.2 \mathrm{~m}$ and the Kitt Peak National Observatory $2.1 \mathrm{~m}$ telescopes. Table 2 summarizes pertinent details of the observing runs, including the dates (col. [1]), the telescope (col. [2]), the imager (col. [3]), and the field of

TABLE 2

\begin{tabular}{|c|c|c|c|}
\hline $\begin{array}{l}\text { UT Date } \\
\text { (1) }\end{array}$ & $\begin{array}{l}\text { Telescope } \\
\text { (2) }\end{array}$ & $\begin{array}{l}\text { IR Imager } \\
\text { (3) }\end{array}$ & $\begin{array}{l}\text { Field of View } \\
\qquad \begin{array}{c}\operatorname{arcsec}) \\
(4)\end{array}\end{array}$ \\
\hline 2000 Mar 13-20 ......................... & $1.2 \mathrm{~m}$ & STELIRCAM & $300 \times 300$ \\
\hline 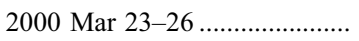 & $2.1 \mathrm{~m}$ & ONIS & $175 \times 350$ \\
\hline 2000 Oct $15-18$ & $2.1 \mathrm{~m}$ & SQIID & $300 \times 300$ \\
\hline 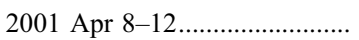 & $1.2 \mathrm{~m}$ & STELIRCAM & $300 \times 300$ \\
\hline 2001 Sep $26-27 \ldots \ldots$ & $1.2 \mathrm{~m}$ & STELIRCAM & $300 \times 300$ \\
\hline 2001 Oct $6-9 \ldots \ldots \ldots$ & $2.1 \mathrm{~m}$ & SQIID & $300 \times 300$ \\
\hline 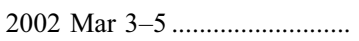 & $1.2 \mathrm{~m}$ & STELIRCAM & $300 \times 300$ \\
\hline 2001 Oct $21-2002$ Mar $23 \ldots \ldots$. & $1.2 \mathrm{~m}$ & STELIRCAM & $300 \times 300$ \\
\hline
\end{tabular}

Journal of OBSERVATIONS 
TABLE 3

BGK00 Infrared Photometry

\begin{tabular}{|c|c|c|c|c|c|c|c|}
\hline $\begin{array}{c}\mathrm{J} 2000.0 \\
\text { Designation } \\
\text { (1) }\end{array}$ & $\begin{array}{c}L \\
(\operatorname{arcsec}) \\
(2)\end{array}$ & $\begin{array}{c}w \\
(\operatorname{arcsec}) \\
(3)\end{array}$ & $\begin{array}{l}\text { P.A. } \\
\text { (deg) } \\
(4)\end{array}$ & $\begin{array}{c}J \\
(5)\end{array}$ & $\begin{array}{l}H \\
(6)\end{array}$ & $\begin{array}{c}K \\
(7)\end{array}$ & $\begin{array}{c}\text { Comments } \\
\text { (8) }\end{array}$ \\
\hline 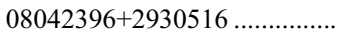 & 38.4 & 3.0 & 90.0 & 14.12 & 13.54 & 13.16 & \\
\hline $08042496+2930236 \ldots \ldots \ldots \ldots \ldots$ & 4.0 & 3.0 & 90.0 & 13.28 & 12.60 & 12.34 & \\
\hline $08065208+1844155 \ldots \ldots \ldots \ldots \ldots$ & 16.8 & 3.0 & 90.0 & 12.53 & 11.82 & 11.58 & \\
\hline 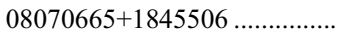 & 28.3 & 3.0 & 90.0 & 14.90 & 14.28 & 14.03 & \\
\hline $08111348+2512249 \ldots \ldots \ldots \ldots \ldots$ & 12.2 & 3.0 & 90.0 & 13.53 & 12.84 & 12.58 & \\
\hline $08111591+2510459 \ldots \ldots \ldots \ldots \ldots$ & 28.8 & 3.0 & 90.0 & 13.87 & 13.22 & 13.03 & \\
\hline $08112548+0853382 \ldots \ldots \ldots \ldots \ldots$ & 26.4 & 3.0 & 90.0 & 14.87 & 14.38 & 14.05 & \\
\hline 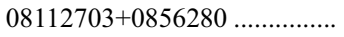 & 28.8 & 3.0 & 90.0 & 14.32 & 13.62 & 13.41 & \\
\hline $08184909+2113053 \ldots \ldots \ldots \ldots \ldots$ & 19.1 & 3.0 & 90.0 & 14.69 & 14.01 & 13.84 & \\
\hline 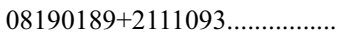 & 4.3 & 3.0 & 90.0 & 15.31 & 14.58 & 14.35 & \\
\hline
\end{tabular}

Notes.-Table 3 is published in its entirety in the electronic edition of the Astronomical Journal. A portion is shown here for guidance regarding its form and content.

view (col. [4]). Each galaxy observation consists of five to nine $60 \mathrm{~s}$ exposures, dithered by $30^{\prime \prime}-75^{\prime \prime}$ in right ascension and declination to allow for accurate sky subtraction and the elimination of bad pixels during data reduction. All sky observations of 10-14 Elias et al. (1982) and United Kingdom Infrared Telescope photometric standards each clear night yielded accurate calibration constants and extinction corrections.

Our approach to reducing the IR camera data is based on previous experience with crowded Galactic fields with extended emission from reflection nebulae and $\mathrm{H}$ II regions (e.g., Barsony et al. 1997; Whitney et al. 1997; Gómez \& Kenyon 2001; Bragg \& Kenyon 2002; Balog et al. 2004). Using a pipeline developed by W. Wyatt, we calibrate each frame with standard routines in NOAO IRAF. ${ }^{2}$ To generate flat fields for each night, we median-filter all program frames using IMCOM, remove hot and dead pixels with a bad pixel mask, and normalize the median of the flat field to unity. After dividing linearized program frames by the flat field, we sort images by their median sky levels and select 11-15 images with sky levels closest to the median sky level of each program field. Medianfiltered images of each set of 11-15 images, scaled by their median levels, yield sky frames for each program field. Skysubtracted images with bad pixels removed have a median background level of zero and noise levels of 20.0-21.0 mag arcsec ${ }^{-2}$ at $H$.

The technique to derive sky frames has several advantages over traditional methods. In ideal conditions, where the sky background varies slowly and monotonically through the night, our approach yields sky frames composed of images acquired close in time to the program frame, as in standard reduction packages. When the sky background fluctuates erratically, our sky frames have fewer low-level background features than traditional sky frames and do not require an offset to match background levels. Comparisons with traditional sky flats show that our approach reduces photometric errors by $0.01-0.02 \mathrm{mag}$.

To construct combined images for each pair, we use DRIZZLE (Fruchter \& Hook 2002) in the STSDAS package within IRAF. Our procedure uses IMEXAM to derive $(x, y)$ centers for each galaxy on each frame, DRIZZLE to shift the images to a common

\footnotetext{
${ }^{2}$ IRAF is distributed by the National Optical Astronomy Observatory, which is operated by the Association of Universities for Research in Astronomy, Inc., under contract to the National Science Foundation.
}

center, and IMCOM to construct a median-filter image of each galaxy from the set of DRIZZLED images.

We derive broadband magnitudes using a simple FORTRAN program to sum the flux in a rectangular aperture that replicates the size and orientation of the slit used for spectroscopic observations ( $(2.5)$. The program uses the $(x, y)$ coordinates of the peak intensity to center the slit on each galaxy. Tests indicate uncertainties of $0.01-0.02 \mathrm{mag}$ for \pm 1 pixel $\left(0.3-1{ }^{\prime \prime} \cdot 2\right)$ uncertainties in the slit position and \pm 1 pixel uncertainties in the size of the slit.

We estimate photometric uncertainties for the survey from repeat measurements. The uncertainty in the photometric calibration for each night is $0.01-0.03 \mathrm{mag}$. Multiple measurements of each galaxy within an observing run yield a typical uncertainty of \pm 0.03 mag for galaxies with $H=13-15$. Repeat measurements acquired on different observing runs indicate a similar uncertainty. The median offset between different observing runs is $\pm 0.02 \mathrm{mag}$. We thus conclude that the typical photometric uncertainty in our slit magnitudes is $\sim 0.04 \mathrm{mag}$.

To estimate the uncertainty in the colors from our slit magnitudes, we derive colors assuming a fixed slit position for all three bands. Repeat measurements of galaxies throughout a single observing run and between observing runs yield identical color uncertainties of \pm 0.04 mag in the $J-H$ and $H-K$ colors. This error estimate is smaller than the \pm 0.06 mag uncertainty expected from simply adding the errors of individual bands in quadrature. However, we determine the color at a fixed slit position for all three bands. Thus, the error in the slit position should not be included in the error in color. We therefore reduce the error in the color by removing the uncertainty in slit position relative to the spectroscopic aperture, $\left[2(0.02)^{2}\right]^{1 / 2} \approx$ $0.03 \mathrm{mag}$, and by the median offset in the photometric calibration between observing runs, $\sim 0.02 \mathrm{mag}$, which yields an expected error, $\approx 0.04$ mag, that agrees with the measured uncertainty.

Table 3 lists the measurements. Column (1) is the J2000.0 galaxy coordinate; column (2) is the slit length, $L$, in arcseconds; column (3) is the slit width, $w$, in arcseconds,; column (4) is the slit position angle in degrees; and columns (5)-(7) are the corresponding $J, H$, and $K$ slit magnitudes, respectively. For nine galaxies without optical spectra (marked with an asterisk in col. [8] of Table 3), we adopted the median slit length, $20^{\prime \prime}$, and the typical position angle of $90^{\circ}$. The colors of these galaxies span the range of $J-H$ and $H-K$ colors of other galaxies in the sample. 

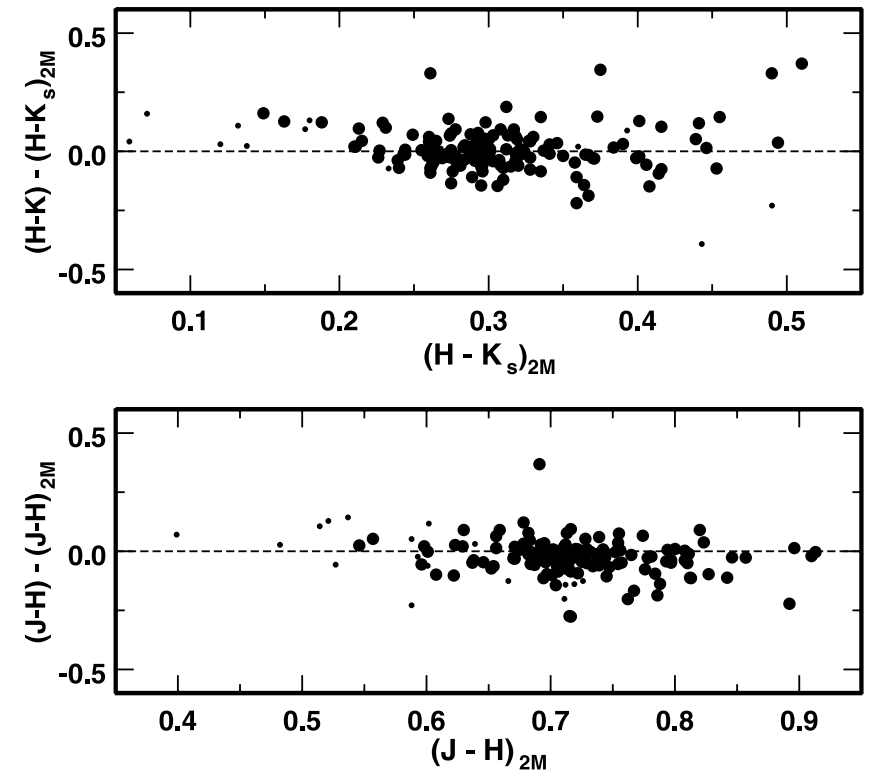

FIG. 1.-Comparison of our slit-aperture $J-H$ and $H-K$ photometry with 2MASS 7 " diameter aperture $\left(H-K_{s}\right)_{2 \mathrm{M}}($ top $)$ and $(J-H)_{2 \mathrm{M}}($ bottom $)$. The small circles represent galaxies with 2MASS isophotal $J>13$. The error in our colors is $0.04 \mathrm{mag}$; the error in the $2 \mathrm{MASS}$ colors is $0.03 \mathrm{mag}$. The dispersion in the relative colors is consistent with these errors.

Figure 1 compares the colors derived from our slit magnitude with 2 MASS colors derived from the 2MASS $7^{\prime \prime}$ diameter magnitudes. We use the 2MASS small-aperture magnitudes here because they are the best available match to our slit magnitudes. The smaller points indicate galaxies with $2 \mathrm{MASS}$ isophotal $J>13$. The mean color difference $\left\langle(J-H)-(J-H)_{2 \mathrm{M}}\right\rangle=$ $-0.03 \pm 0.005 ;\left\langle(H-K)-\left(H-K_{s}\right)_{2 \mathrm{M}}\right\rangle=0.01 \pm 0.005$. Removing the large outliers in Figure 1, the dispersion in $\sigma[(\mathrm{J}-$ $\left.H)-(J-H)_{2 \mathrm{M}}\right]=0.05 \mathrm{mag}$, and $\sigma\left[(H-K)-\left(H-K_{s}\right)_{2 \mathrm{M}}\right]=$ 0.05 mag. The typical errors in the 2MASS colors are 0.03 mag: $\sigma\left[(J-H)-(J-H)_{2 \mathrm{M}}\right]$ and $\sigma\left[(H-K)-\left(H-K_{s}\right)_{2 \mathrm{M}}\right]$ are thus consistent with the 0.04 mag error we estimate in our slit-aperture photometry. These measures refer to the ensemble of points in the plots. We suspect that the small blueward offset of our $J-H$ relative to 2MASS results from differences in the aperture; our extracted apertures tend to be somewhat larger than the 2MASS $7^{\prime \prime}$ aperture. The reddest outliers in $(H-K)-\left(H-K_{s}\right)_{2 \mathrm{M}}$ occur because our broader $K$ filter provides greater sensitivity to emission from hot dust ${ }^{3}$ (see Hunt et al. [2002] and $\S \S 3.1$ and $3.2)$.

\subsection{IRAS Data}

We downloaded $12-100 \mu \mathrm{m}$ photometry from the IRAS extended and point-source catalogs using the IRSA Web interface (Beichman et al. 1985). These data yield 246 matches to pair galaxies, with 156 systems having both reliable 2MASS and $60 \mu \mathrm{m}$ photometry. All but 10 of these 156 systems have reliable $100 \mu \mathrm{m}$ fluxes. In five pairs, IRAS unambiguously detected both galaxies; in 47 pairs, IRAS detected one of the galaxies unambiguously; and in 107 pairs, the IRAS detection is unresolved.

Table 4 lists sample IRAS data for 10 BGK00 pairs of galaxies. We include a J2000.0 BGK00 coordinate designation (col. [1]), IRAS B1950.0 coordinate designation (col. [2]), and the IRAS photometry (cols. [3]-[6]). The typical error in the $60 \mu \mathrm{m}$ detections is $0.25 \mathrm{Jy}$; in the $100 \mu \mathrm{m}$ detections it is $0.5 \mathrm{Jy}$. The typical error in the upper limits at $60 \mu \mathrm{m}$ is $0.5 \mathrm{Jy}$. In this table we associate the $I R A S$ flux with the pair galaxy closest to the IRAS coordinate. In our discussion we treat the IRAS emission as a property of the system; the poor spatial resolution of $I R A S$ prevents robust association with individual galaxies.

The sample of pairs resolved by IRAS (SSM04) offers some insight into the completeness of the IRAS identifications we make in the BGK00 sample. The BGK00 sample contains all pairs in a magnitude-limited redshift survey with projected separations $<50 h^{-1} \mathrm{kpc}$; the SSM04 pairs are restricted to systems separated by fewer than three galaxy diameters. Interestingly, the BGK00 sample contains four pairs within the 5.24 Jy limit of the SSM04 sample, but they are too widely separated to meet the SSM04 diameter criterion. Table 5 lists these pairs: column (1) gives the $\mathrm{J} 2000.0$ coordinate of the BGK00 galaxy closest to the IRAS source, column (2) gives the IRAS B1950.0 identification, and columns (3)-(6) give the $I R A S$ fluxes.

\footnotetext{
${ }^{3}$ With a full width at half-maximum of $0.4 \mu \mathrm{m}(2-2.4 \mu \mathrm{m})$, the standard $K$ filter is $25 \%$ wider than the $K_{s}$ filter, which has a full width at half-maximum of $0.3 \mu \mathrm{m}(2-2.3 \mu \mathrm{m})$. We acquired our $K$ data in dry conditions, where $K$ is more sensitive than $K_{s}$ to hot-dust emission with $T=600-1000 \mathrm{~K}$.
}

TABLE 4

IRAS Mid-InFrared Photometry

\begin{tabular}{|c|c|c|c|c|c|}
\hline \multirow{2}{*}{$\begin{array}{c}\mathrm{J} 2000.0 \\
\text { Designation } \\
(1)\end{array}$} & \multirow{2}{*}{$\begin{array}{c}I R A S \\
\text { DESIGNATION } \\
\text { (2) }\end{array}$} & \multicolumn{4}{|c|}{ Flux (Jy) } \\
\hline & & $\begin{array}{c}12 \mu \mathrm{m} \\
(3)\end{array}$ & $\begin{array}{c}25 \mu \mathrm{m} \\
\text { (4) }\end{array}$ & $\begin{array}{c}60 \mu \mathrm{m} \\
\quad(5)\end{array}$ & $\begin{array}{c}100 \mu \mathrm{m} \\
(6)\end{array}$ \\
\hline $08042396+2930516 \ldots \ldots \ldots \ldots \ldots \ldots$ & $08012+2939$ & $0.25 \mathrm{~L}$ & $0.37 \mathrm{~L}$ & 0.43 & $1.10 \mathrm{~L}$ \\
\hline $08042496+2930236 \ldots \ldots \ldots \ldots \ldots \ldots$ & $08012+2939$ & $0.25 \mathrm{~L}$ & $0.37 \mathrm{~L}$ & 0.43 & $1.10 \mathrm{~L}$ \\
\hline 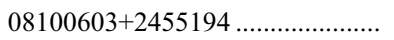 & $08070+2503$ & $0.48 \mathrm{~L}$ & $0.31 \mathrm{~L}$ & 0.93 & 1.13: \\
\hline $08101117+2453344 \ldots \ldots \ldots \ldots \ldots \ldots \ldots \ldots$ & $08070+2503$ & $0.48 \mathrm{~L}$ & $0.31 \mathrm{~L}$ & 0.93 & 1.13: \\
\hline $08111348+2512249 \ldots \ldots \ldots \ldots \ldots \ldots$ & $08082+2521$ & $0.25 \mathrm{~L}$ & $0.46 \mathrm{~L}$ & 2.14 & 5.97 \\
\hline $08111591+2510459 \ldots \ldots \ldots \ldots \ldots \ldots$ & $08082+2521$ & $0.25 \mathrm{~L}$ & $0.46 \mathrm{~L}$ & 2.14 & 5.97 \\
\hline 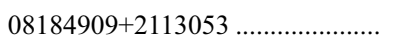 & $08161+2120$ & $0.25 \mathrm{~L}$ & $0.61 \mathrm{~L}$ & $0.54:$ & 1.51 \\
\hline $08190189+2111093 \ldots \ldots \ldots \ldots \ldots \ldots . .$. & $08161+2120$ & $0.25 \mathrm{~L}$ & $0.61 \mathrm{~L}$ & $0.54:$ & 1.51 \\
\hline $08194129+2202311 \ldots \ldots \ldots \ldots \ldots \ldots$ & $08168+2211$ & $0.25 \mathrm{~L}$ & $0.34 \mathrm{~L}$ & 0.74 & 2.12 \\
\hline 08194833+2201531 …………........ & $08168+2211$ & $0.25 \mathrm{~L}$ & $0.34 \mathrm{~L}$ & 0.74 & 2.12 \\
\hline
\end{tabular}

Notes.-Table 4 is published in its entirety in the electronic edition of the Astronomical Journal. A portion is shown here for guidance regarding its form and content. To preserve associations of IRAS detections with pair galaxies, we list IRAS associations for each galaxy in the pair. This listing duplicates flux measurements for pair galaxies for which $I R A S$ could not spatially resolve the pair. For the IRAS fluxes, "L" indicates an upper limit (quality flag 1), and a colon indicates an approximate flux (quality flag 2). 
TABLE 5

BGK00 Pairs Brighter than Surace Flux Limits

\begin{tabular}{cccccc}
\hline \hline $\begin{array}{c}\text { Pair ID } \\
(1)\end{array}$ & $\begin{array}{c}F_{12} \\
(2)\end{array}$ & $\begin{array}{c}F_{25} \\
(3)\end{array}$ & $\begin{array}{c}F_{60} \\
(4)\end{array}$ & $\begin{array}{c}F_{100} \\
(5)\end{array}$ & $\begin{array}{c}\text { (Jy) } \\
(6)\end{array}$ \\
\hline $011934.70+032445.22 \ldots \ldots \ldots .$. & $01171+0308$ & $0.56:$ & 1.10 & 6.41 & 12.24 \\
$023726.83+210716.52 \ldots \ldots \ldots .$. & $02345+2053$ & 0.56 & 1.22 & 10.18 & 16.93 \\
$091549.24+405355.59 \ldots \ldots \ldots \ldots$. & $09120+4107$ & 0.52 & 1.07 & 8.75 & 16.60 \\
$160513.04+203230.75 \ldots \ldots \ldots .$. & $16030+2040$ & $0.24:$ & 0.87 & 7.04 & 10.10 \\
\hline
\end{tabular}

In most of the SSM04 pairs, the galaxies are too faint at $B$ to be included in BGK00. However, 33 of the SSM04 pairs lie within the limits defined by the selection of the BGK00 sample. Among these, two pairs are missing from the BGK00 sample because the galaxies were not resolved in the original Zwicky et al. (1968) catalog on which the redshift survey was based. Unresolved pairs at small angular separation are a limiting factor for all samples of close pairs. All told, the BGK00 and SSM04 samples have 31 pairs in common and differ by a total of 6 pairs in the range of complete overlap.

As we did for the BGK00 pairs, we also extracted 2MASS magnitudes for the SSM04 pairs. We use these data only to show that the SSM04 pairs include more extreme dusty objects than the BGK00 sample. The Appendix compares further basic IRAS properties of the BGK00 and SSM04 pairs. More detailed comparison of these pair samples is beyond the scope of this paper because of the complex selection of the SSM04 pairs and because we do not have spectroscopy for them.

\subsection{Spectroscopy}

BGK00 and BGK03 describe the spectroscopic observations and data reduction in detail. Here we briefly review the procedures we used.

We made observations of 502 galaxies with the FAST spectrograph at the $1.5 \mathrm{~m}$ Tillinghast telescope on Mount Hopkins. We observed each galaxy for $\sim 10-20$ minutes through a $3^{\prime \prime}$ wide slit and used a 300 line $\mathrm{mm}^{-1}$ grating to disperse the light over 4000-7000 ̊..

Our spectra are representative of the "central" region of each galaxy. The apertures extracted from the flat-fielded data range in length from 1".74 to 29 "'.7, corresponding to $0.25-13.7 h^{-1} \mathrm{kpc}$ with a mean of $2.4 h^{-1} \mathrm{kpc}$ (see Table 4). The measured equivalent widths are the ratio of the flux in the line to the surrounding continuum corrected for Balmer absorption. We estimate the amount of absorption by taking the maximum absorption equivalent width in $\mathrm{H} \delta$ or $\mathrm{H} \gamma$ and then adding that equivalent width to both $\mathrm{H} \alpha$ and $\mathrm{H} \beta$. Table 1 of BGK03 lists the $\mathrm{EW}(\mathrm{H} \alpha)$ and $\mathrm{H} \alpha / \mathrm{H} \beta$ for the galaxies in our sample. The typical error in the $\mathrm{EW}(\mathrm{H} \alpha)$ is $10 \%$; in the Balmer decrement the error is typically $20 \%$. Repeat measurements suggest that the error results largely from uncertainty in the slit position.

We remove active galactic nuclei (AGNs) and "ambiguous" objects that may be AGN-dominated from both the BGK00 sample and the NFGS by using the theoretical optical classification scheme developed by Kewley et al. (2001a). There are three such objects in the NFGS (Kewley et al. 2002); there are 32 in the BGK00 spectroscopic sample. Because the number of these objects is small, removal has a negligible effect on the analyses below; we remove the objects for consistency in our focus on star formation. Among the 265 galaxies without spectra, we expect about 17 AGN and/or "ambiguous" galaxies, a residual number too small to impact any trends in the data set.
In any case, the mean $J-H$ of the BGK00 AGN and ambiguous galaxies is $0.02 \mathrm{mag}$ bluer and their $H-K$ is $0.02 \mathrm{mag}$ redder than the 470 non-AGN galaxies in the spectroscopic sample.

\section{THE NEAR-INFRARED PROPERTIES OF GALAXY PAIRS}

Although the NIR colors of galaxies span a narrow range, they provide an interesting window on star-forming galaxies. In this section we explore the IR properties of the BGK00 sample. Even though the BGK00 sample is $B$-selected, the sample is nearby enough that the pairs can be resolved in 2MASS, and all of the galaxies are detected in 2MASS.

The NIR probes a complex combination of stellar population, reddening, and gaseous and thermal dust emission. We use our own IR photometry and spectroscopy to probe these issues. For this exploration our photometry has two advantages over 2MASS: (1) we extract photometry in the aperture in which we have spectroscopy, and (2) we use the standard $K$ filter, which extends to longer wavelength than the $2 \mathrm{MASS} K_{s}$. The $K$ filter is more sensitive to thermal emission from $600-1000 \mathrm{~K}$ dust.

\subsection{The Near-Infrared Color-Color Diagram}

The NIR colors for a normal unreddened stellar population span a small range in $J-H$ and $H-K_{S}$ (Aaronson 1977; Giuricin et al. 1993). By collecting data from two sets of close pairs selected either by optical morphology or by projected separation and line-of-sight relative velocity, Giuricin et al. concluded that "interacting" galaxies display normal $J-H$ colors and redder $H-K$ colors than a "normal" galaxy population. Making use of the existing $L$-band data, they also concluded that the redder $H-K$ colors indicate the presence of thermal emission from hot $600-1000 \mathrm{~K}$ dust. Here we compare the $J-H$ and $H-K$ colors for the BGK00 sample of close pairs with the NFGS sample of normal galaxies and with the SSM04 sample of IRASselected pairs.

We have assembled data for much larger sets of both pairs and normal galaxies than were considered by Giuricin et al. (1993) and Aaronson (1977), respectively. The errors in the IR colors for galaxies in the Giuricin et al. (1993) sample are typically 0.1 in $J-H$ and 0.06 in $H-K$, substantially exceeding the errors in our data. The sample considered by Giuricin et al. (1993) is an exhaustive compilation of inhomogeneous IR data from the literature, but the sample is not complete in any band. The pair sample we consider is a complete $B$-limited sample with NIR photometry for all objects. We examine the sample in the broader context of a similarly selected sample of normal galaxies from the NFGS and the FIR-selected sample of SSM04. In all cases, the NIR data are uniformly acquired from 2MASS.

Figure 2 shows color-color diagrams for the 2MASS $J H K_{s}$ data (top left) and for our JHK data (top right) for the individual galaxies in the BGK00 pairs. To set the BGK00 sample in a broader context, we also show 2MASS data for the individual galaxies in the SSM04 pairs (bottom left) and for the NFGS sample of normal galaxies. The $K$-corrections for galaxies in these samples are small, typically $\lesssim 0.03$ at $K_{s}$ and $\lesssim 0.01$ at $J$ and $H$ (Poggianti 1997). In the NFGS panel (bottom right) the curves show how the colors of solar neighborhood stars on the main sequence (solid curve) and giant branch (dot-dashed curve) bracket the normal galaxy colors (Bessell \& Brett 1988).

In all panels in Figure 2 the line with bars shows the contribution at $K$ from thermal emission by dust with a temperature of $1000 \mathrm{~K}$; each bar marks a $10 \%$ increase in the hot-dust 

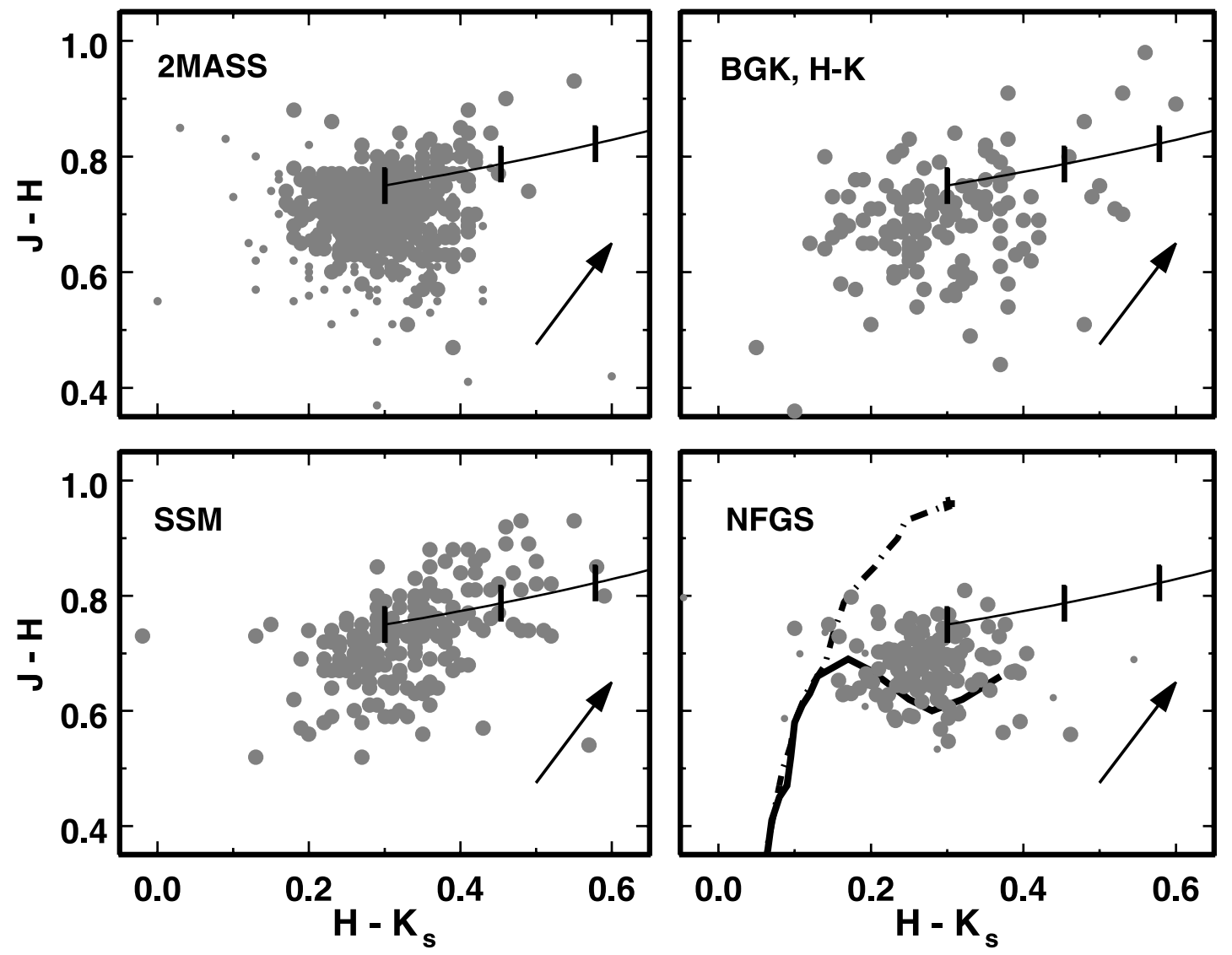

FIG. 2.-NIR color-color diagrams for the BGK00 sample 2MASS data (top left), the BGK00 small aperture JHK data (top right; this paper), the SSM04 pair 2MASS data (bottom left), and the NFGS normal galaxy 2MASS data (bottom right). Note the large spread of colors in the pair samples relative to the NFGS. The small circles denote galaxies with $J>13$; these objects tend to be blue as a result of the original $B$-selected sample. In each panel the arrow shows the reddening vector, and the curve with bars shows the fractional contribution of $1000 \mathrm{~K}$ dust at $K$; each bar marks a $10 \%$ increment. In the NFGS panel, the curves show the color of main-sequence stars (thick solid line) and the giant branch (dot-dashed line) from Bessel \& Brett (1988).

contribution. The arrow indicates the reddening vector (Bessell \& Brett 1988).

The NFGS sample of normal optically selected galaxies spans the expected narrow range of NIR colors. Kolmogorov-Smirnov (K-S) tests show that the probability that the $J-H$ and $H-K_{S}$ distributions for the BGK00 samples and the NFGS are drawn from the same parent distributions is $1.6 \times 10^{-12}$ and $8.5 \times 10^{-10}$, respectively. Both the mean $J-H$ and $H-K$ are slightly redder in the BGK00 sample than in the NFGS, and the spread in both colors is larger, consistent with the results of Cutri \& McAlary (1985). Giuricin et al. (1993) were unable to see the difference in the distribution of $J-H$ for pairs in their smaller sample.

In our sample of pair galaxies the NIR colors scatter both toward red $J-H$ and $H-K$ and toward blue $J-H$ and $H-K$ more than the NFGS. As we discuss below, this behavior results from the presence of young central bursts of star formation that scatter the colors blueward and from dusty bursts that scatter the colors redward. The original selection of the sample at $B$ and subsequent "observation" in the NIR favors detection of the blueward scatter relative to the detection of dusty bursts. Selection in a redder band favors the redward scatter relative to the blueward scatter introduced by relatively unobscured bursts; we demonstrate this point by examining the SSM04 IRAS-selected pairs.

As expected, the SSM04 sample, selected at $60 \mu \mathrm{m}$, shows a notable extension toward red $H-K_{s}$, much more impressive than in our $B$-selected data set. Because we do not have complete spectroscopy for this sample, we cannot remove the AGNs; however, it is improbable that all of the reddest objects in $H-K_{S}$ are AGNs. There are 16 known AGNs in the SSM04 sample (Veilleux et al. 1995; Kewley et al. 2001b; Corbett et al. 2003). These AGNs have mean NIR colors $J-H=0.74 \pm 0.02$ and $H-K_{s}=0.33 \pm 0.01$, consistent with the overall mean for the sample. Only one of these AGNs is a red outlier in $H-K_{s}$. Based on the properties of the known AGNs, we expect only two to four red outliers in $H-K_{s}$, even if $30 \%$ of the SSM04 galaxies are AGNs. The well-populated extension toward red $H-K_{s}$ is thus very probably a property of star-forming objects.

Without longer wavelength data, particularly at $L$, there is also an ambiguity in the relative contributions of reddening and dust emission for star-forming objects with $0.4<H-K_{s}<0.5$. However, even in the narrow $K_{s}$ filter, it is essentially impossible to explain the full extension of the colors of star-forming galaxies in the SSM04 sample along the dust emission track in Figure 2 by any other straightforward mechanism (Carico et al. 1986; Hunt et al. 2002).

In our $B$-selected pairs sample, the bluest galaxies are generally faint in the NIR; these objects also tend to have the lowest intrinsic $B$-band luminosities in the pairs sample. Many of these objects have $J>13$ and thus appear as small points in the colorcolor diagram. They tend to appear at blue $J-H$ with a range of $H-K_{s}$. It is interesting that the SSM04 sample, which includes nearby intrinsically low-luminosity objects, also includes pair 

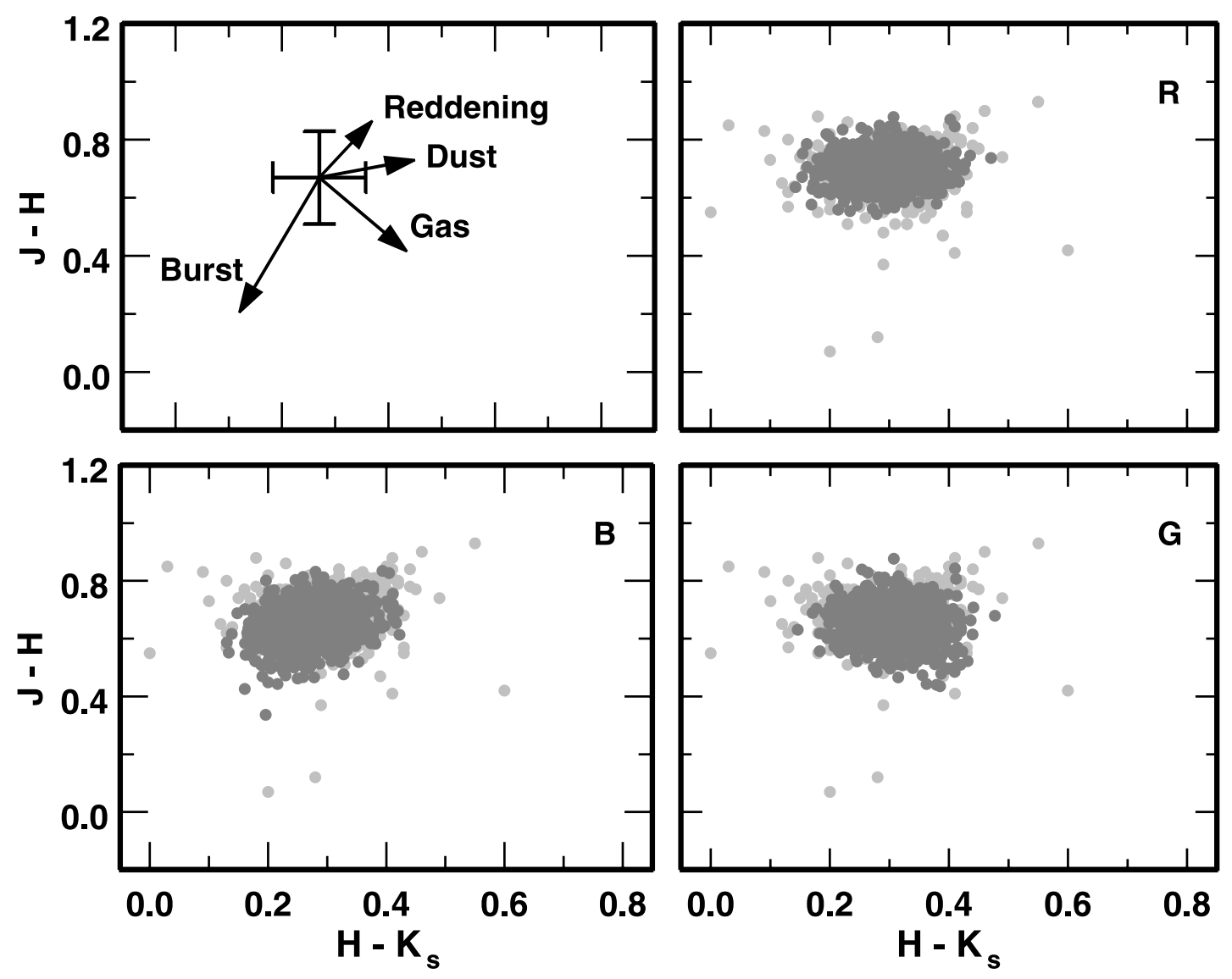

FIG. 3.-Heuristic statistical model for the 2MASS BGK00 pairs color-color diagram. The top left panel is a schematic showing the physical components of the model. The cross indicates the colors of a normal galaxy population. The arrows show the direction a galaxy moves in this color space as a result of reddening, hot-dust emission, gaseous emission, and young bursts as indicated. In the other three panels light gray circles show the data, and dark gray circles show the model. In the top right panel the model (R; Table 6) contains only the scatter in colors and reddening, in the bottom left panel we add bursts of star formation emission (B; Table 6), and in the bottom right panel we add gaseous emission (G; Table 6). Table 6 gives the model parameters. In each panel, note the change in shape of the central mass of circles and the behavior of the outliers. The models account for most of the observed spread of colors.

galaxies with these bluer $J-H$ colors. Overall, the larger range of NIR colors of pairs compared with the NFGS is independent of the inclusion of these fainter objects, although they accentuate the difference in the expected sense. The Appendix contains a more extensive comparison of the properties of the SSM04 and BGK00 samples.

Figure 2 (top right) shows our $J H K$ slit-aperture photometry for a subset of $160 \mathrm{BGK} 00$ galaxies. More objects in this subset extend toward redder $J-H$ and $H-K$ than in the 2MASS BGK00 color-color diagram (top left). There are two reasons for this extension. Although color gradients are generally small in the IR, some objects are significantly redder in their central regions (Griersmith et al. 1982; Glass \& Moorwood 1985; Devereux 1989; Bushouse \& Werner 1990; Carico et al. 1990; Jarrett 2000; Kewley et al. 2005). The broader $K$ filter is also more sensitive to hot-dust emission, which has a negligible effect on $J-H$ but produces a redder $H-K$. We discuss these $J H K$ colors in more detail below.

To understand the difference between the color-color diagrams for the BGK00 pair sample and the NFGS sample of normal galaxies, we construct a simple model. The model is intended to account qualitatively for the spread of colors rather than to explain details of individual cases. The model has six ingredients: (1) an estimate of the mean color for a normal galaxy population, (2) a spread in color that represents both the intrinsic spread of a normal population and the measurement error,
(3) reddening, (4) emission from a young stellar population associated with a burst of star formation, (5) gaseous emission from $\mathrm{H}$ II regions, and (6) thermal reradiation from hot dust. The schematic in Figure 3 shows the normal range of NIR colors (error bars), along with arrows indicating the direction of the effect of the various contributors to the NIR colors.

To reconstruct the distribution of colors for galaxies in the BGK00 sample, we begin with the median colors of the NFGS: $(J-H)_{0}=0.67$ and $\left(H-K_{s}\right)_{0}=0.27$. The mean $E_{B-V}$ for the NFGS in our luminosity range is $0.28 \pm 0.03$. The median NFGS colors are completely consistent with earlier studies (see, e.g., Aaronson 1977; Giuricin et al. 1993).

To account for the spread of colors in a normal galaxy population and for the measurement error in the colors, we first add a dispersion of $0.05 \mathrm{mag}$ in both colors, denoted by $\sigma_{J-H}$ and $\sigma_{H-K}$ in Table 6. With a typical $2 \mathrm{MASS}$ color error of $0.03 \mathrm{mag}$, the intrinsic dispersion in color we assume is $0.04 \mathrm{mag}$, consistent with the results of Aaronson (1977). We select a random additional reddening by taking the absolute value of a Gaussian with a dispersion of 0.020 in the extinction at $H-K_{s}, E_{H-K_{s}}$. We denote this contribution by $\sigma_{R}$ in Table 6 . The extinction at $J-H, E_{J-H}$, is $1.95 E_{H-K_{\mathrm{s}}}$. The dispersion $\sigma_{R}$ corresponds to an additional $A_{V}=0.3$ (Bessell \& Brett 1988) and moves the colors redward along the reddening vector. Figure 3 (top right; model R) shows that this simple prescription accounts for most of the range and for the redward shift in the color-color diagram 
TABLE 6

JHK Model Parameters

\begin{tabular}{|c|c|c|c|c|c|c|c|c|}
\hline $\begin{array}{l}\text { Model Number } \\
\text { (1) }\end{array}$ & $\begin{array}{c}(J-H)_{0} \\
\text { (2) }\end{array}$ & $\begin{array}{c}(H-K)_{0} \\
\text { (3) }\end{array}$ & $\begin{array}{c}\sigma_{J-H} \\
\text { (4) }\end{array}$ & $\begin{array}{c}\sigma_{H-K} \\
\text { (5) }\end{array}$ & $\begin{array}{l}\sigma_{B} \\
(6)\end{array}$ & $\begin{array}{l}\sigma_{D} \\
\text { (7) }\end{array}$ & $\begin{array}{l}\sigma_{G} \\
(8)\end{array}$ & $\begin{array}{l}\sigma_{R} \\
(9)\end{array}$ \\
\hline R & 0.67 & 0.27 & 0.05 & 0.05 & 0.00 & 0.000 & 0.00 & 0.020 \\
\hline В & 0.67 & 0.27 & 0.05 & 0.05 & 0.05 & 0.000 & 0.00 & 0.020 \\
\hline 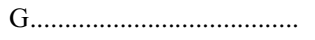 & 0.67 & 0.27 & 0.05 & 0.05 & 0.00 & 0.000 & 0.05 & 0.020 \\
\hline 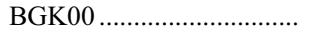 & 0.72 & 0.20 & 0.06 & 0.06 & 0.08 & 0.008 & 0.04 & 0.06 \\
\hline
\end{tabular}

for the BGK00 pairs, but outliers remain, particularly toward the bluest $J-H$ and reddest $H-K_{s}$.

We can account statistically for most of the outliers by including contributions from young stellar populations, free-free emission, and dust. Models B and $\mathrm{G}$ explore the effect of adding a burst of star formation and gaseous emission, respectively. Table 6 gives the parameters for these models: column (1) gives the model designation; columns (2) and (3) list the fiducial colors, $(J-H)_{0}$ and $(H-K)_{0}$; columns (4) and (5) give the normal spread of $J-H$ and $H-K$ colors, respectively; and columns (6) (9) give the contributions from a young burst, hot dust, gaseous emission, and reddening, respectively. The models are not unique; they are intended to demonstrate the impact of the various contributions to the NIR emission and to show that reasonable parameters account for the observed spread of colors.

Model B in the bottom left panel of Figure 3 shows model R with the addition of a burst contribution. In this example the burst color is $J-H=H-K_{s}=0$, characteristic of A stars. For this demonstration we ignore the complexities of variation in the initial mass function, metallicity, and spread in age and duration of the bursts. We select the fractional contribution of the burst at $H$ by taking the absolute value of a Gaussian with a dispersion of 0.05 ; we denote this contribution by $\sigma_{B}$ in Table 6 . This choice is consistent with the BGK03 optical analysis of burst strengths. For the $40 \%-60 \%$ burst strengths most common in the BGK03 sample, a 5\% burst contribution at $H$ corresponds to a roughly $50 \%$ contribution at $R$ for the typical $R-H \sim 2.5$ we measure in the slit aperture for the bluer objects. Addition of a "burst" moves the colors blueward in both $J-H$ and $H-K_{s}$.

Model $\mathrm{G}$ in the bottom right panel of Figure 3 shows model $\mathrm{R}$ with the addition of gaseous emission with a color of $J-H=0$ and $H-K_{s}=0.7$ (Campbell \& Terlevich 1984; Whitelock 1985; Ramos-Larios \& Phillips 2005). The addition of gaseous emission moves the colors blueward in $J-H$ and redward in $H-K_{s}$. We select the fractional contribution of the gaseous emission at $H$ by taking the absolute value of a Gaussian with a dispersion of 0.08 and denote this contribution by $\sigma_{G}$ in Table 6 .

Figure 3 shows that our heuristic model accounts for the colors of all but a few extreme outliers. The outliers with blue $H-K_{s}$ and red $J-H$ are fainter than $J=13$ and may have large errors in their colors. The two very blue objects in $J-H$ may be dominated by a strong burst and/or gaseous emission. The reddest object in both $J-H$ and $H-K_{S}$ requires at least a $10 \%$ contribution from hot dust emission at $K_{s}$.

For the BGK00 sample, our small-aperture broader $K$-band data underscore the necessity of accounting for emission from hot dust in modeling the interaction. These conclusions are in accord with Giuricin et al. (1993). In the model shown in Figure 4, $(J-H)_{0}=0.72$ and $(H-K)_{0}=0.20$; the error in $J-H$ and $H-K$ is 0.06 mag, corresponding to a color error of $0.04 \mathrm{mag}$ and an intrinsic spread in color of $0.045 \mathrm{mag}$. We draw the ad- ditional reddening from a Gaussian with a dispersion of 0.06 in $H-K$, the fractional contribution from gaseous emission at $H$ is drawn from a Gaussian with a dispersion of 0.04 , and the fractional burst contribution at $H$ is drawn from a Gaussian with a dispersion of 0.08 . To match the extension toward the reddest $H-K$, we introduce a contribution from dust emission. The fractional dust emission contribution at $H$ is drawn from a Gaussian with a dispersion of 0.008 and denoted by $\sigma_{D}$ in Table 6. At $K$, this dispersion is 0.09 . Thus, the dust contribution for the reddest objects in $H-K$ is $10 \%-20 \%$ at $K$.

Figure 5 shows images of a few of the objects in our IR smallaperture photometry sample with the reddest central $H-K$ colors. For the red objects in the left and middle panels, there is no spectroscopic evidence of AGN activity. We exclude the AGN in the right panel from the sample; its blue companion has an $\mathrm{EW}(\mathrm{H} \alpha)=37 \AA$.

The Infrared Space Observatory provided the first suggestion of hot $600-1000 \mathrm{~K}$ dust in star-forming galaxies (Helou et al. 2000). Subsequent $J H K L^{\prime}$ observations demonstrate that some actively star-forming galaxies have $K-L^{\prime} \geq 1$, consistent with hot-dust emission (Hunt et al. 2002). Hunt et al. (2002) suggest that the hot dust is associated with the intense far-ultraviolet radiation field in compact $(\leq 100 \mathrm{pc})$ regions of active star formation, which might arise naturally from galaxy-galaxy interactions and mergers. In their sample of 26 galaxies, they were unable to detect any correlation between the presence of hot dust and the much cooler dust.

For the BGK00 pairs the ratio of 60 to $100 \mu \mathrm{m} I R A S$ fluxes implies emission from dust at temperatures in the range $20-70 \mathrm{~K}$

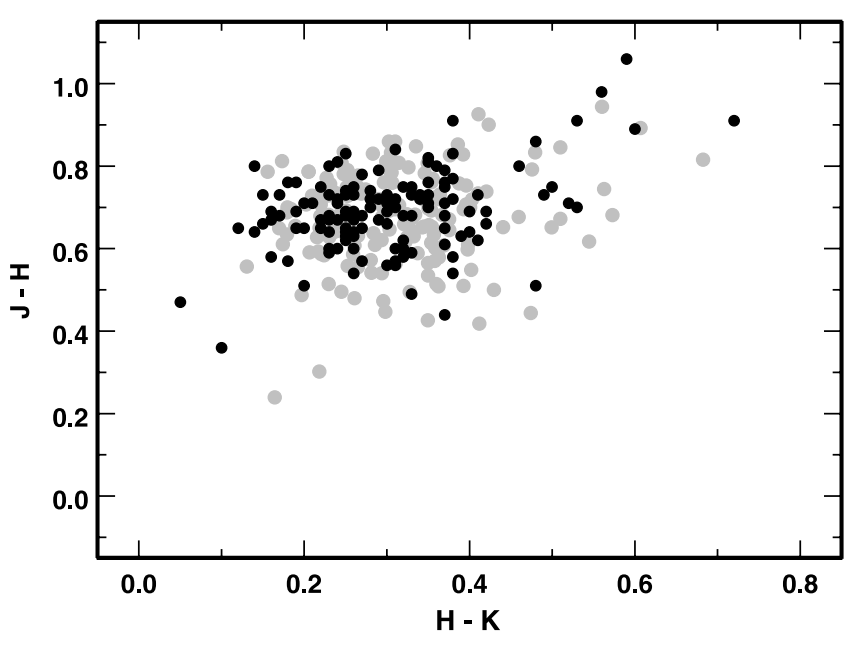

FIG. 4.-Comparison of slit photometry colors for 160 BGK00 galaxies with the model (parameters are in Table 6; BGK00). Gray circles represent the data, and black circles show the model. The $K$ band (as opposed to $K_{s}$ photometry) is more sensitive to hot-dust emission, which is necessary to account for the reddest objects in $H-K$. 


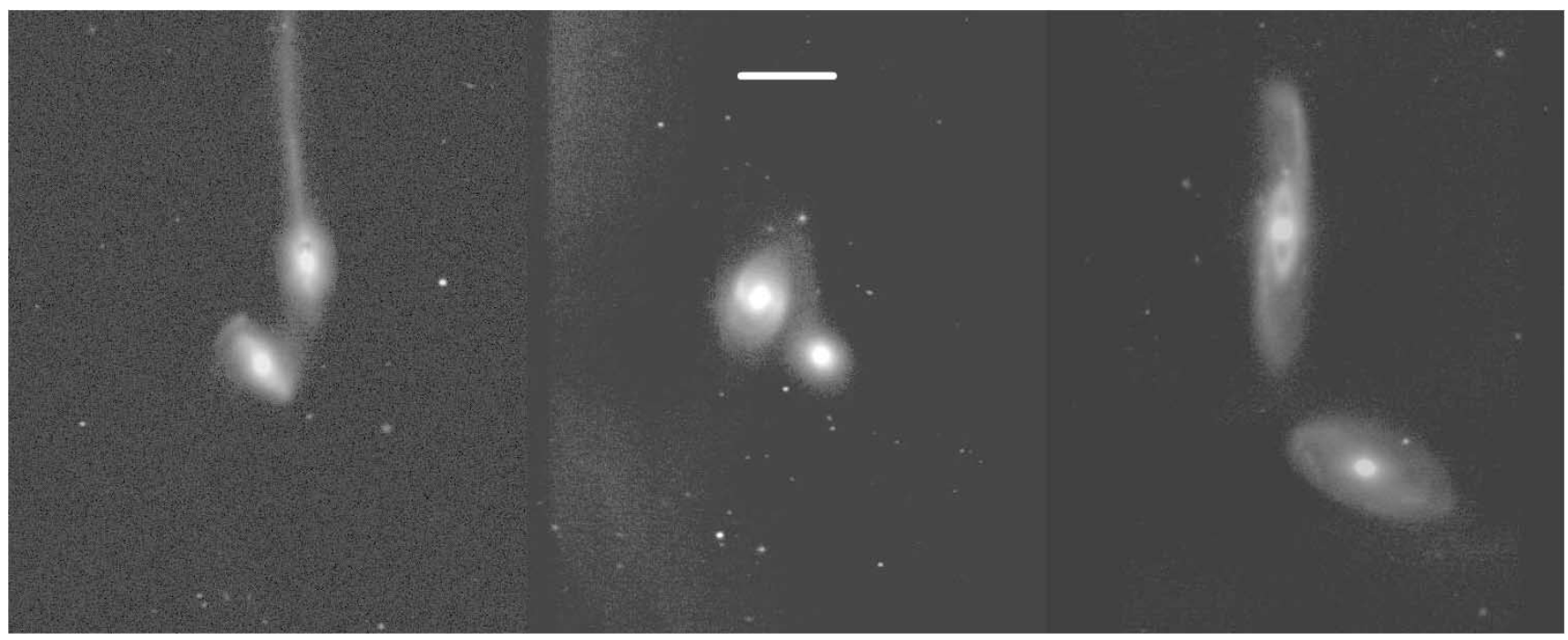

FIG. 5.-NIR images for three galaxy pairs. The images have north up, east to the left, and scales of 0.34 pixel $^{-1}$. Left: $J$-band image of $124344.0+310017.9$ $(H-K=0.60)$ and $124345.3+305943.8(H-K=0.37)$. The northern component has a dust lane on JHK images. Middle: $K$-band image of 152418.9+415040.7 $(H-K=0.28)$ and $152420.9+415059.5(H-K=0.72)$. The redder eastern component has a tidal tail not visible in the Digitized Sky Survey image. Right: $K$-band image of 113704.8+321108.9 $(H-K=0.82)$ and $113707.1+321227.2(H-K=0.22)$. The southern component is a red AGN that we exclude from the sample; the blue northern component, retained in the sample, has a bright ring of star formation. The $30^{\prime \prime}$ white bar gives the image scale.

assuming a blackbody spectrum (Beichman et al. 1985). We do not have enough pairs with both small-aperture photometry and IRAS detections to make a meaningful comparison of $H-K$ with $L_{\mathrm{FIR}} / L_{H}$; we thus use the much larger sample of $2 \mathrm{MASS}$ $H-K_{S}$ data to see whether there is any relationship between the presence of hot-dust and FIR emission from cooler dust.

Figure 6 shows the distribution of the normalized FIR luminosity $F_{\mathrm{FIR}} / F_{H}$ as a function of $H-K_{s}$ for the BGK00 pairs. We define the FIR flux in the standard way (Helou et al. 1988; Sanders \& Mirabel 1996),

$$
F_{\mathrm{FIR}}=1.26 \times 10^{-14}\left(2.58 f_{60}+f_{100}\right) \mathrm{W} \mathrm{m}^{-2},
$$

where $f_{60}$ and $f_{100}$ are the 60 and $100 \mu \mathrm{m}$ fluxes, respectively. If a pair is resolved we sum the fluxes for the components; for each pair the luminosity we plot represents the total for the pair and the $H-K_{s}$ color is the appropriately weighted pair color.

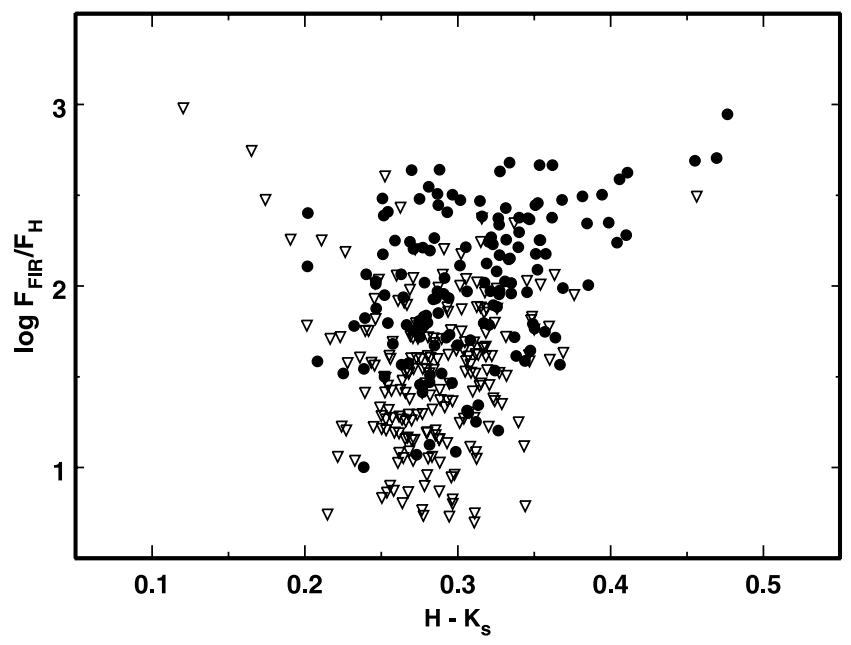

FIG. 6. $-F_{\mathrm{FIR}} / F_{H}$ for the BGK00 pairs. The circles represent detections, and the triangles represent upper limits.
Figure 6 shows $F_{\mathrm{FIR}} / F_{H}$ as a function of $H-K_{s}$ from 2MASS for the BGK00 pairs for both IRAS detections and upper limits. The $F_{\text {FIR }}$ value is proportional to the star formation rate (Kennicutt 1998; Calzetti et al. 2000; Charlot et al. 2002; Kewley et al. 2002), and $F_{H}$ is a measure of the stellar mass. Thus, $F_{\mathrm{FIR}} / F_{H}$ provides a measure of the normalized or specific star formation rate. Figure 6 shows that the reddest $H-$ $K_{s}$ colors correspond to relatively large $F_{\mathrm{FIR}} / F_{H}$. Using the ASURV package (Lavalley et al. 1992), the Spearman rank probability of no correlation including the upper limits in Figure 6 is $<10^{-4}$. The increasing scatter in $H-K_{s}$ with increasing $F_{\mathrm{FIR}} / F_{H}$ is also a striking feature of Figure 6 . At the bluest $H-K_{s}$, all of the points with large $F_{\mathrm{FIR}} / F_{H}$ are upper limits (triangles); nearly all of the reddest $H-K_{s}$ are detections at large $F_{\mathrm{FIR}} / F_{H}$. The data suggest that the reddest galaxies in $H-K_{s}$ contain dust emitting over a wide temperature range. These data indicate that, as suggested by Hunt et al. (2002), some galaxies that undergo strong central bursts of star formation and thus have substantial specific star formation rates contain compact star-forming complexes with very red $H-K_{s}$. These regions may be similar to the dusty super star clusters found in interacting galaxies and ULIRGs (e.g., Guiderdoni et al. 1998; Gallagher \& Smith 1999; Bekki \& Couch 2001; Keel \& Borne 2003; Kassin et al. 2003).

Emission from hot dust may also be associated with AGN activity. We have removed the spectroscopically identifiable AGNs from our sample. However, we show in the next section that the reddest objects in $H-K$ have weak or even undetectable $\mathrm{H} \alpha$ emission. It is possible that AGN activity is hidden. Because AGNs are rare in the large sample we can classify spectroscopically, we favor vigorous star formation as the explanation of the reddest $H-K$ colors. Hard X-ray observations with Chandra and mid-IR imaging with Spitzer would be useful in better identifying AGNs (Alonso-Herrero et al. 2006).

It is frustrating that in Figure 6 the poor resolution of IRAS prevents plotting quantities for individual galaxies that can be readily identified with points in the color-color diagrams. Clearer tests of the underlying physics that dominates the colorcolor plots requires the resolution of Spitzer. Hopefully, large 

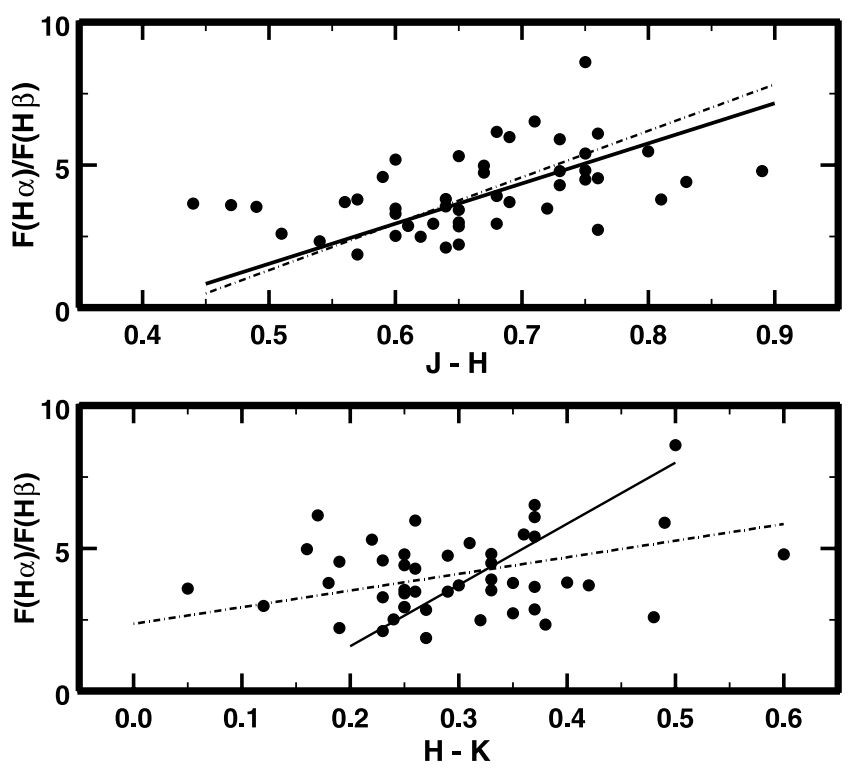

FIG. 7.-Balmer decrement for 45 BGK00 galaxies as a function of $J-H$ (top) and $H-K$ (bottom). The colors are measured in the spectroscopic aperture. The solid lines show the Galactic reddening law, and the dot-dashed lines show the best-fit slope. For $J-H$ the fitted slope is essentially the same as the Galactic reddening law; for $H-K$ the slopes differ because hot-dust emission affects the $H-K$ color

objectively selected samples of nearby pairs with Spitzer observations will soon be forthcoming.

\subsection{Spectroscopy and Infrared Colors}

We next use our spectroscopy to elucidate further the physics that underlies the color-color diagrams. We combine the spectroscopy with our $J H K$ photometry in the spectroscopic aperture. Figure 7 shows the relationship between the Balmer decrement and the $J-H$ color (see Moorwood et al. [1987] for the first demonstration of this particular correlation but without a fitted slope). With the exception of a few outliers, it is remarkable that these objects, some of which contain young or heavily reddened bursts of star formation, have essentially the same reddening law as the Galaxy. The Spearman rank probability of no correlation is $10^{-4}$ and the best-fit slope $\left(16.2 \pm 1.9, \chi^{2} /\right.$ dof $=2.1$; dotdashed line) is close to the Galactic reddening law (14.0; solid line; Bessel \& Brett 1988).

A large $\mathrm{EW}(\mathrm{H} \alpha)$ indicates a strong burst of star formation. BGK03 conclude that these bursts can account for $40 \%-60 \%$ of the galaxy light at $R$. These substantial bursts should result in bluer than normal NIR colors. Figure 8 shows the $J-H$ color as a function of $\mathrm{EW}(\mathrm{H} \alpha)$. Indeed, at the very bluest colors $(J-H \lesssim 0.57), 55 \%$ of the galaxies have $\mathrm{EW}(\mathrm{H} \alpha) \gtrsim 25 \AA$ and $9 \%$ have $\mathrm{EW}(\mathrm{H} \alpha) \lesssim 10 \AA$. At $J-H \gtrsim 0.78,11 \%$ of the galaxies have $\operatorname{EW}(\mathrm{H} \alpha) \gtrsim 25 \AA$ and $56 \%$ have $\operatorname{EW}(\mathrm{H} \alpha) \lesssim 10 \AA$. The Spearman rank probability of no correlation is $6.8 \times 10^{-5}$. The $\mathrm{EW}(\mathrm{H} \alpha)=0$ objects have a major impact on the Spearman rank probability; eliminating them gives a probability of 0.11 of no correlation.

Figure 7 shows that the Balmer decrement is less well correlated with $H-K$; the Spearman rank probability of no correlation is 0.26 . We see little correlation here because dust emission affects $H-K$ but not $J-H$ (see the schematic in Fig. 2 and the model in Fig. 3, which requires dust emission to account for the data). The dot-dashed line indicates the best-fit slope

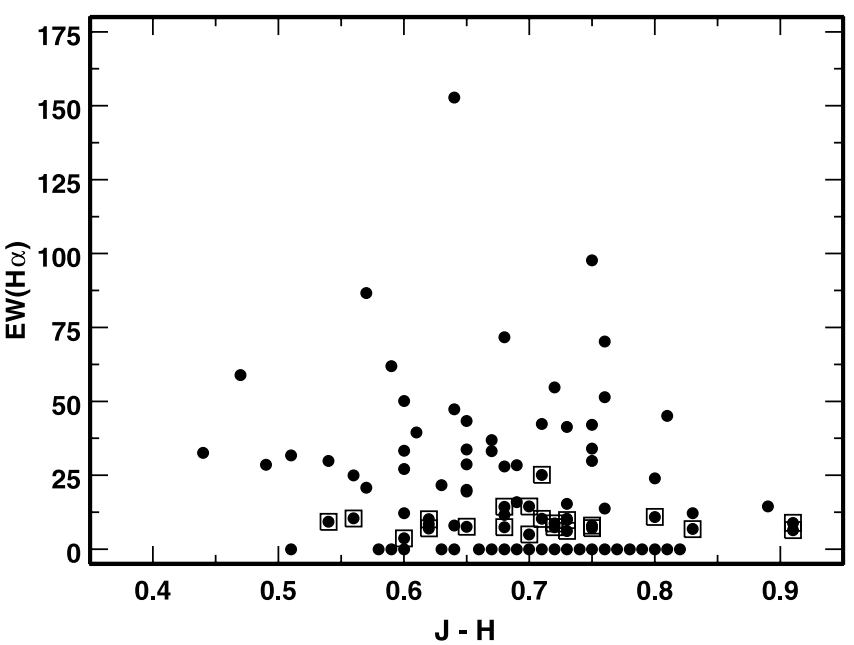

Fig. 8.-Equivalent width of $\mathrm{H} \alpha$ as a function of $J-H$ for 151 BGK00 galaxies. The color is measured in the spectroscopic aperture. Boxes denote galaxies with nonzero $\mathrm{EW}(\mathrm{H} \alpha)$ but undetectable $\mathrm{H} \beta$.

$5.8 \pm 0.4$, with an abysmal $\chi^{2} /$ dof $=41$. The solid line shows the slope of the standard Galactic reddening law (21.4; Bessell \& Brett 1988).

Figure 9 shows the relationship between $\mathrm{EW}(\mathrm{H} \alpha)$ and $H-K$. The apparent correlation is not significant; the Spearman rank probability of no correlation is 0.65 . The largest $\mathrm{EW}(\mathrm{H} \alpha)$ values occur at bluer $H-K \lesssim 0.35 ; 10 \%$ of galaxies with $H-K \lesssim$ 0.35 have $\mathrm{EW}(\mathrm{H} \alpha) \gtrsim 50 \AA$. None of the reddest galaxies $(H-$ $K \gtrsim 0.45)$ have $\operatorname{EW}(\mathrm{H} \alpha) \gtrsim 50 \AA$. Although most of the reddest galaxies have $\operatorname{EW}(\mathrm{H} \alpha) \lesssim 10 \AA$, there are also objects at blue $H-K$ with $\mathrm{EW}(\mathrm{H} \alpha)=0$.

In Figures 8 and 9, boxed symbols indicate galaxies with nonzero $\mathrm{EW}(\mathrm{H} \alpha)$ but without a measurable $F(\mathrm{H} \alpha) / F(\mathrm{H} \beta)$. Of the five objects with $H-K>0.5$ (these objects are the most likely to contain dusty compact regions of intense star formation), two have $\mathrm{EW}(\mathrm{H} \alpha)=0$, two have immeasurable Balmer decrements, and one has $F(\mathrm{H} \alpha) / F(\mathrm{H} \beta) \sim 5$. All of these objects are in IRAS-detected systems with $L_{\mathrm{FIR}} / L_{H} \gtrsim 1.8$. The member galaxies thus may contain dust-enshrouded bursts. Accounting for these "hidden" bursts is important for a full picture of tidally

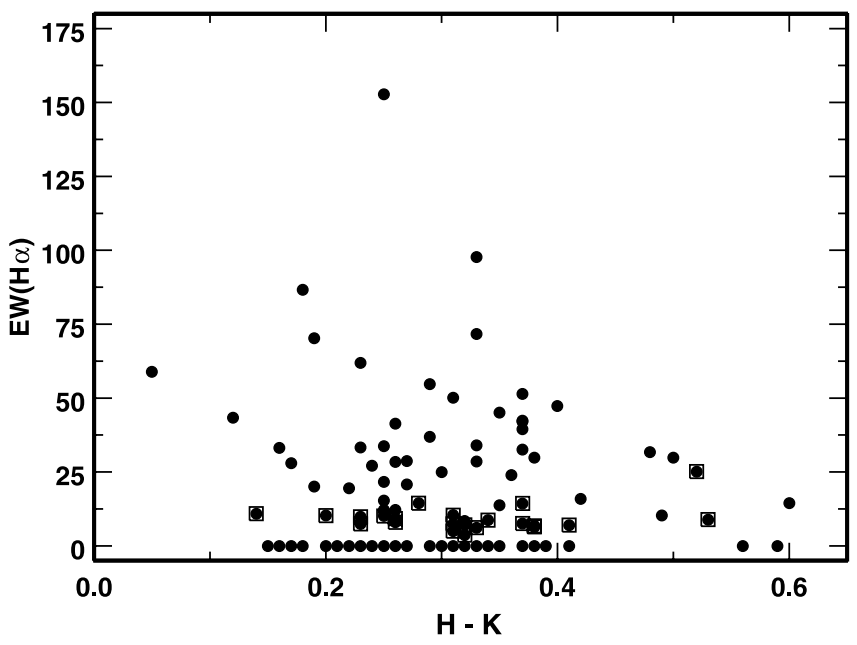

FIG. 9.-Equivalent width of $\mathrm{H} \alpha$ as a function of $H-K$ for $151 \mathrm{BGK} 00$ galaxies. The color is measured in the spectroscopic aperture. Boxes denote galaxies with nonzero $\mathrm{EW}(\mathrm{H} \alpha)$ but undetectable $\mathrm{H} \beta$. 

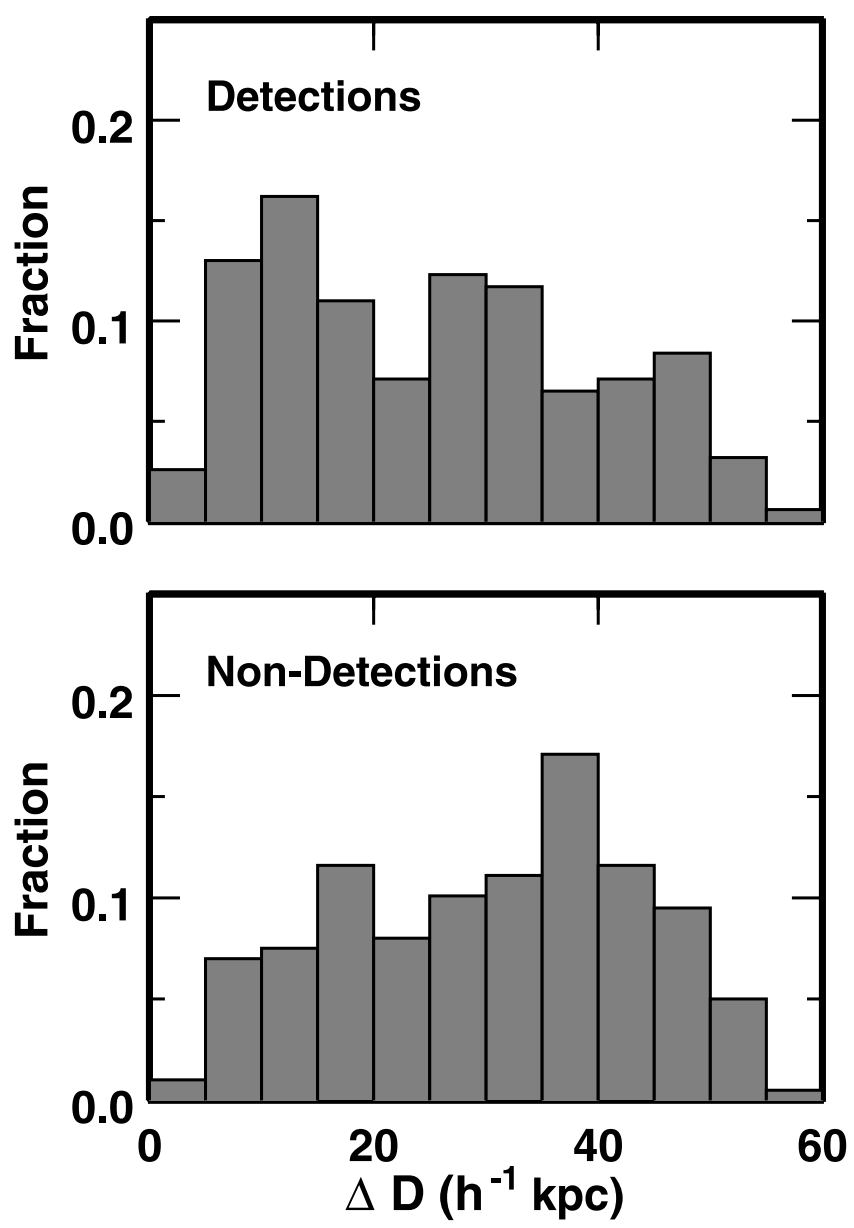

FIG. 10.-Normalized distribution of projected separations for $I R A S$-detected BGK00 pairs (top) and undetected pairs (bottom).

triggered star formation and for calculations of the star formation rate density throughout the universe.

\section{THE INFRARED AND TRIGGERED STAR FORMATION}

In this section we investigate the relationship between the IR properties of the BGK00 pairs and the projected separation of the pair, a measure of the interaction. An impressive range of star formation indicators are correlated with the projected separation, including the $\mathrm{EW}(\mathrm{H} \alpha)(\mathrm{BGK} 00$; BGK03), the star formation birth-rate parameter for pairs in the Two Degree Field survey (Lambas et al. 2003; Alonso et al. 2004), the mean specific star formation rate derived from Sloan Digital Sky Survey (SDSS) $\mathrm{H} \alpha$ and $z$-band luminosities (Nikolic et al. 2004), the concentration index measured in the $r$ band as a measure of the presence of nuclear bursts of star formation (Nikolic et al. 2004), and metallicity as an indicator of gas infall in the BGK00 pairs (Kewley et al. 2006).

\subsection{Far-Infrared Specific Star Formation Rates}

Figure 10 shows the normalized distribution of projected separations for pairs in the BGK00 sample with and without IRAS detections. The K-S probability that the two distributions are drawn from the same population is $3.3 \times 10^{-4}$. The pairs detected in IRAS have a median separation of $20 h^{-1} \mathrm{kpc}$; the median for the undetected pairs is $32 h^{-1} \mathrm{kpc}$. These distri-

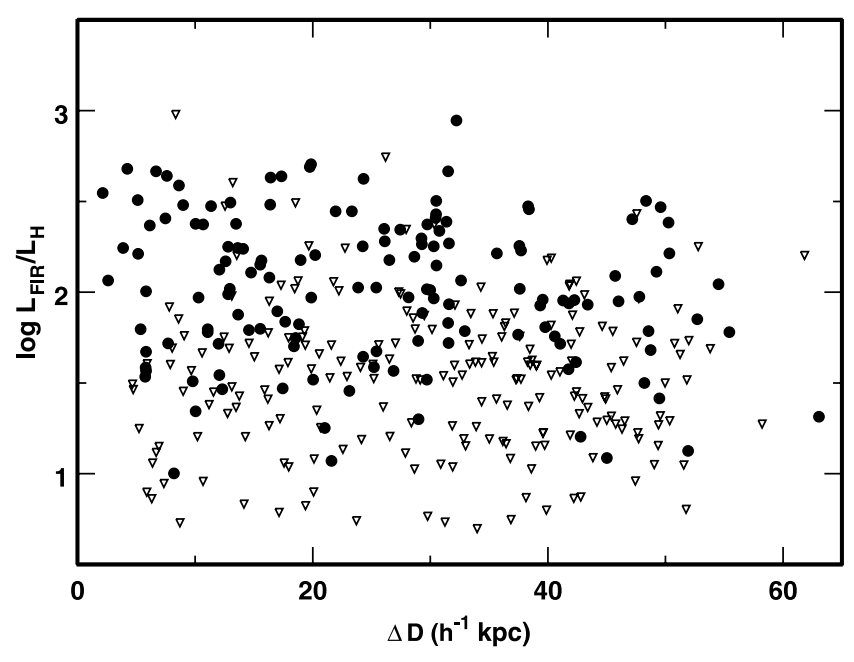

FIG. 11. $-L_{\mathrm{FIR}} / L_{H}$ as a function of separation for the BGK00 pairs. Circles represent detections, and triangles represent upper limits. Note that $L_{\mathrm{FIR}} / L_{H}$ refers to individual pairs, not individual galaxies.

butions suggest that pairs that are most probably close together in space have greater specific star formation rates.

To explore the connection between specific star formation rate and $\Delta D$ further, we calculate $\log \left(L_{\mathrm{FIR}} / L_{H}\right)$ (Fig. 11, circles) or the upper limit on $\log \left(L_{\mathrm{FIR}} / L_{H}\right)$ (Fig. 11, triangles) for each BGK00 pair. Figure 11 shows the distribution of $\log \left(L_{\mathrm{FIR}} / L_{H}\right)$, a proxy for the specific star formation rate, as a function of $\Delta D$ for pairs with $\Delta D<60 h^{-1} \mathrm{kpc}$.

To examine the correlation between specific star formation rate and $\Delta D$ we use the ASURV package (Lavalley et al. 1992), which treats the upper limits. The Spearman rank probability of no correlation is $<10^{-4}$. Smaller $\Delta D$ favors larger $\log \left(L_{\mathrm{FIR}} / L_{H}\right)$.

For comparison with Figure 11, Figure 12 shows $\mathrm{EW}(\mathrm{H} \alpha)$ as a function of $\Delta D$ for the entire BGK00 sample. In contrast with Figure 11, where the IRAS resolution limits us to plotting fluxes for systems, we plot $\mathrm{EW}(\mathrm{H} \alpha)$ for individual galaxies in Figure 12.

Nikolic et al. (2004) discuss the normalized FIR star formation rate as a function of separation for a subset of their SDSS pairs. They consider only IRAS detections and discuss the inherent

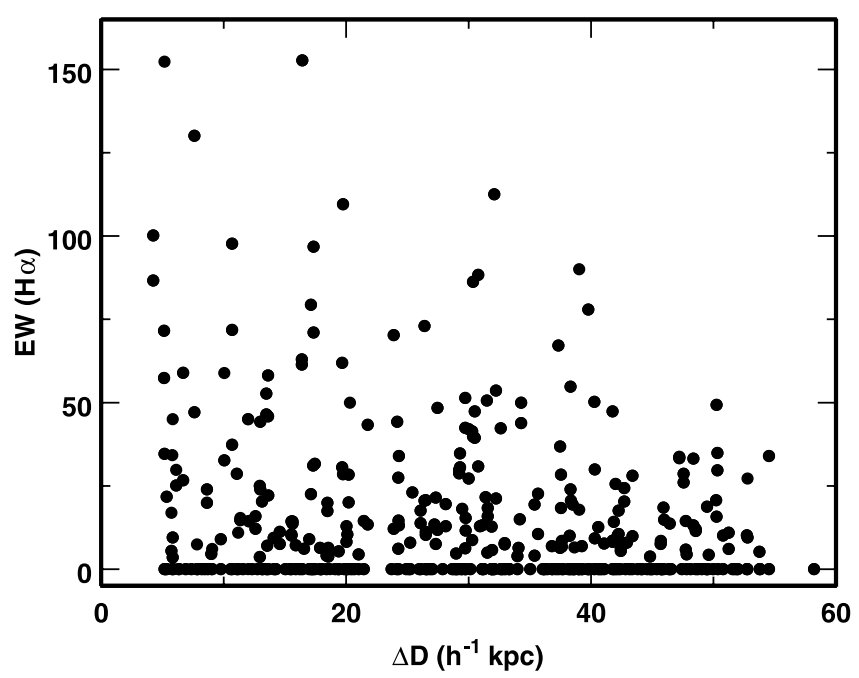

FIG. 12.- $\mathrm{EW}(\mathrm{H} \alpha)$ as a function of projected separation for BGK00 pairs. Each circle represents an individual galaxy. 


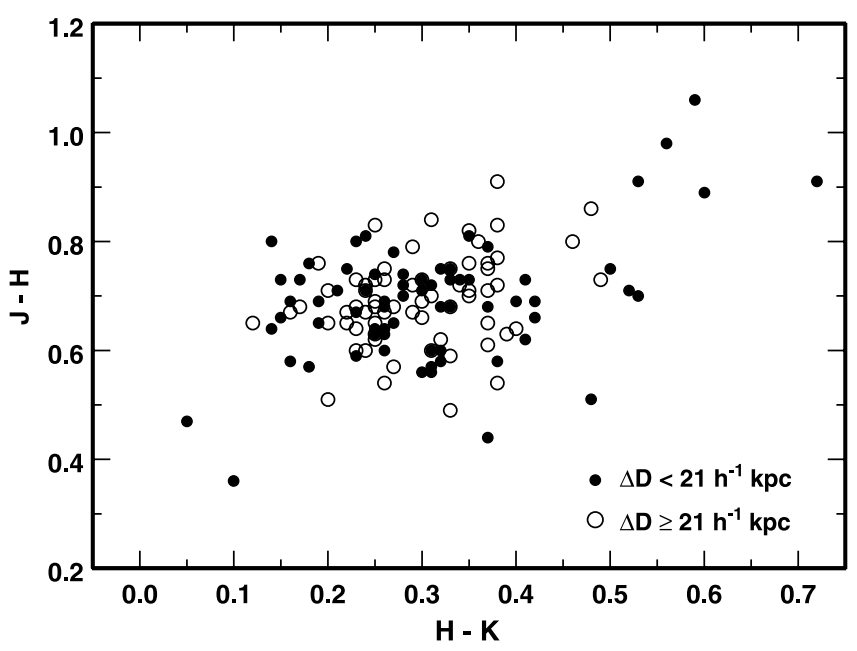

FIG. 13. - Small-aperture photometry NIR color-color diagram for 160 galaxies in BGK00 pairs with projected separations $\Delta D<21 h^{-1} \mathrm{kpc}$ ( filled circles) and with projected separation $\Delta D \geq 21 h^{-1} \mathrm{kpc}$ (open circles).

bias in that approach. They conclude that the normalized star formation rates calculated from $\mathrm{H} \alpha$ emission or from FIR fluxes decrease with increasing projected separation in agreement with our conclusion derived from both detections and upper limits. A serious limitation on comparison of the spectroscopic and FIR indicators of the star formation rate is that our spectroscopic measurements are limited to a central aperture; the IRAS fluxes rarely even resolve the pair.

BGK00 and BGK03 plot the relation in Figure 12 for subsets selected by local density. Like Nikolic et al. (2004) and other investigators, we make no density selection here. The Spearman rank probability that the data are uncorrelated is $<10^{-4}$, consistent with the result based on the IRAS fluxes and upper limits for the same sample.

The data provide strong evidence for a relationship between the normalized star formation rate and the projected separation of the pair regardless of the measure we use for the star formation rate. It would be fascinating to see a similar plot for galaxies in pairs with complete spectroscopy observed with Spitzer so that a more direct comparison could be made between the star formation rate indicators for a large objectively selected pair sample.

\subsection{Near-Infrared Colors}

The situation in the NIR is complex. Dust emission produces a redder $H-K_{s}$ but leaves $J-H$ essentially unchanged. Reddening produces generally redder NIR colors, and a burst of star formation produces bluer colors. Gaseous emission produces bluer $J-H$ and redder $H-K$. Because of the multiplicity of sources of NIR emission and because the full range of NIR colors of stellar populations is small, the NIR colors alone are not useful for estimating the strength of a burst of star formation.

The NIR color-color diagram (Fig. 2) shows that the distribution of NIR colors of close pairs differs from a sample of normal galaxies. For a more complete model, $L$-band and resolved $60 \mu \mathrm{m}$ photometry are necessary to separate the effects of reddening from the effects of dust emission. Nonetheless, the NIR colors indicate a possibly interesting dependence on the projected separation of the pair.

Figure 13 shows the NIR color-color diagrams for the slitaperture $J H K$ sample divided at the median separation, $\Delta D=$
$21 h^{-1} \mathrm{kpc}$. There are 80 galaxies in each of the two subsamples. The two subsamples appear different to the eye in the sense that the spread in colors, particularly $H-K$, is larger at smaller separation; all of the bluest and reddest objects at $H-K$ are at smaller projected separations. There are outliers in $H-K$ toward the blue (indicating central bursts of star formation) and toward the red (indicating dusty bursts). It is interesting that the outliers in the small $\Delta D$ sample are in the directions indicated in the schematic of Figure 3 for young burst and dust emission contributions. All of the candidate dust-enshrouded bursts with $H-K>0.5$ are in pairs with small projected separation. However, a two-dimensional K-S test shows that the samples have a $10 \%$ probability of being drawn from the same underlying distribution.

Figure 13 suggests that the range of colors may be greater for tighter pairs, but the strength of the conclusion may be limited by the sample size. The 2MASS data also yield insignificant differences in color distribution with projected separation, but the 2MASS aperture is large and the 2MASS $K_{s}$ is less sensitive to the effects of dust. Exploration of a larger sample of smallaperture data would be worthwhile, especially with the addition of $L$-band data. There is potential for detecting the impact of both dusty bursts and blue bulges in this approach.

\section{CONCLUSION}

We use the BGK00 sample of pairs of galaxies selected from the complete CfA2 redshift survey to examine NIR and FIR photometry for clues to the nature of the galaxy-galaxy interaction. The depth of the CfA2 survey is well-matched to 2MASS, and all of the nearly 800 galaxies in the sample have 2MASS photometry. The sample of close pairs we analyze is much larger than those considered in previous investigations of the IR properties of close pairs.

We use a combination of 2MASS photometry, deep JHK photometry in small apertures, spectroscopy, and IRAS data to explore the IR as a probe of triggered bursts of star formation. We find the following:

1. The distribution of $J-H, H-K_{S}$, and $H-K$ colors of pairs is broader than the corresponding distribution for a sample of normal galaxies. We interpret this difference as evidence for bursts of star formation that produce an extension toward bluer colors and for a combination of reddened and/or dusty bursts that produce an extension toward redder colors, particularly in $H-K$. In the color-color diagram the reddest objects in $H-K$ are also red in $J-H$. The colors of these objects follow a track for thermal emission from $600-1000 \mathrm{~K}$ dust. The reddest $H-K$ colors require emission from hot dust.

2. A statistical model including emission from a young stellar population, gaseous emission from $\mathrm{H}$ in regions, and emission from hot dust explains the outliers in IR color-color diagrams. This model shows that triggered central bursts affect the NIR colors. The effects are complex because of the multiplicity of emission processes important in the NIR.

3. We use our spectroscopy and small-aperture photometry to show that the central $J-H$ colors and Balmer decrements are consistent with the Galactic reddening law. However, $H-K$ colors are essentially uncorrelated with the Balmer decrement as a result of emission from hot dust.

4. We identify a set of objects with central $H-K \geq 0.45$, $F_{\mathrm{FIR}} / F_{H} \gtrsim 1.8$, and small $\mathrm{EW}(\mathrm{H} \alpha)$. We argue that these objects harbor compact dust-enshrouded bursts possibly similar to the super star clusters identified in well-studied interacting galaxies. These objects may be examples of "hidden" bursts or, possibly, 
"hidden" AGNs. Their presence supports the contention that $\mathrm{H} \alpha$ surveys underestimate the volume-averaged star formation rate in the nearby universe.

5. We examine measures of the specific star formation rate as a function of the projected separation of the pairs in our sample. Both spectroscopic and FIR photometric measures show that the specific star formation rate decreases with increasing projected separation. The poor spatial resolution of IRAS prevents direct comparison of these measures.

6. Examination of the NIR color-color diagram as a function of projected separation shows that all of the outliers indicative of a central burst of star formation lie in pairs at small separation. Although our sample is too small to make the case for a statistically significant dependence of the NIR color-color diagram on projected separation, the data suggest that larger samples of deep, small-aperture NIR photometry would be a basis for identifying both the very blue and very red central bursts. An increase in the dispersion might be expected as a result of triggered bursts that produce blue central regions in the absence of dust and very red central regions in the presence of dust.

Taken together, these results indicate that a larger sample of pairs with complete spectroscopy and with small-aperture NIR photometry and resolved mid-IR photometry from Spitzer would be an important data set for isolating the effects of young bursts, reddening, and thermal dust emission over the course of galaxy-galaxy interactions and subsequent mergers.

We thank Jason Surace for providing early versions of the computer-readable tables from SSM04. We thank the anonymous referee for comments that prompted several important clarifications. This paper uses data from the Infrared Astronomical Satellite, a joint project of the Netherlands Agency for Aerospace Programs, the US National Aeronautics and Space Administration, and the UK Science and Engineering Research Council. This publication makes use of data products from the Two Micron All Sky Survey, which is a joint project of the University of Massachusetts and the Infrared Processing and Analysis Center, California Institute of Technology, funded by the National Aeronautics and Space Administration and the National Science Foundation. This research has made use of the NASA/IPAC Infrared Science Archive, which is operated by the Jet Propulsion Laboratory, California Institute of Technology, under contract with the National Aeronautics and Space Administration.

\section{APPENDIX}

\section{IRAS PROPERTIES OF THE BGK00 AND SSM04 SAMPLES}

Differences in the selection of samples of close pairs can have a marked effect on the global properties of the ensemble of galaxies. The BGK00 and SSM04 samples are an interesting case of selection in very different wavelength ranges and with different separation criteria. The BGK00 sample is selected from a complete magnitude-limited redshift survey based on the Zwicky et al. (1968) catalog. As a result of the construction of the Zwicky catalog, the BGK00 sample is deficient in pairs separated by $\leq 5 h^{-1} \mathrm{kpc}$. The SSM04 sample is flux-limited at $60 \mu \mathrm{m}$. The restrictive criterion of projected pairwise separation to three average projected galaxy diameters biases the sample
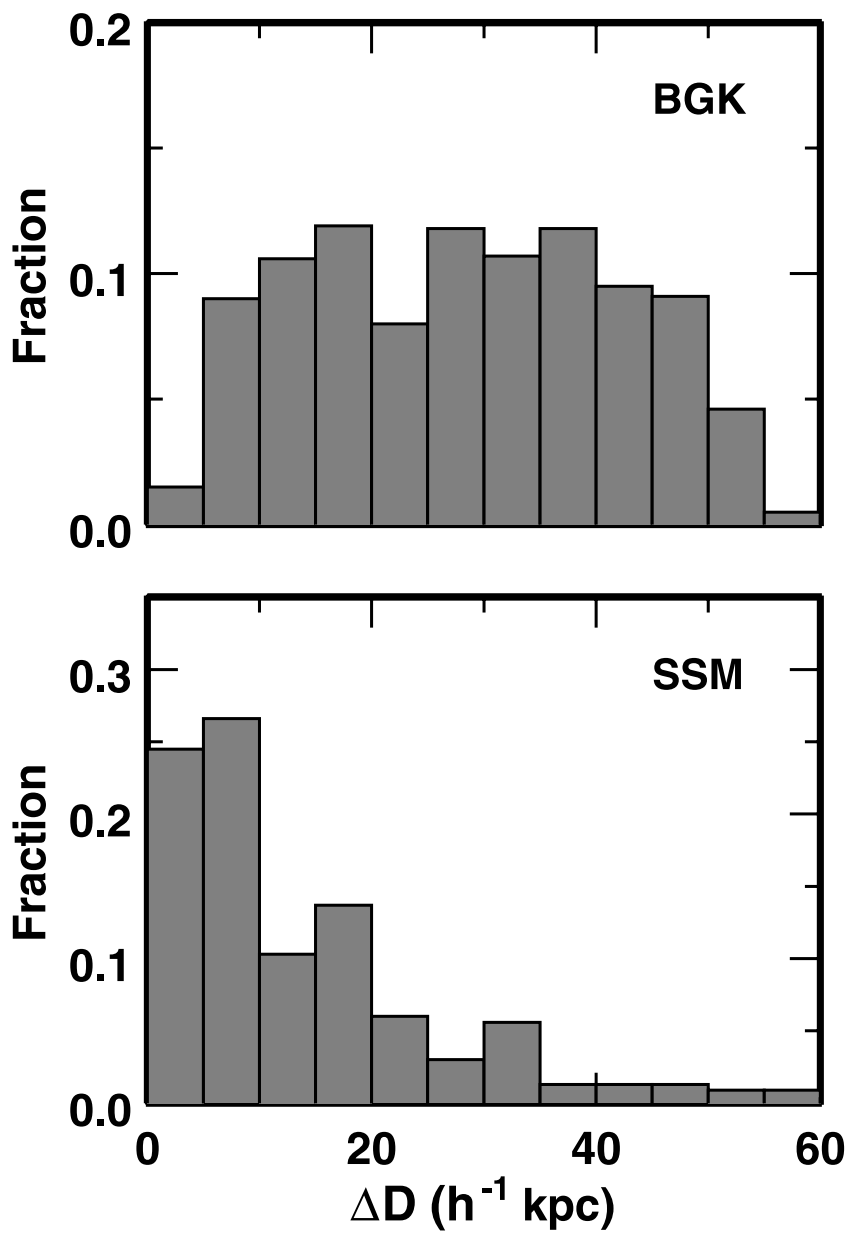

FIG. 14.-Normalized distributions of projected pairwise separations for the BGK00 and SSM04 samples.

against widely separated pairs. Figure 14 shows the difference in the distribution of projected pairwise separations for the two samples.

Figure 15 shows that the SSM04 sample spans a somewhat broader range of $H$-band luminosity, and the BGK00 sample is more narrowly peaked. The mean $c z$ of the SSM04 sample is $4287 \mathrm{~km} \mathrm{~s}^{-1}$; for BGK00 it is $5852 \mathrm{~km} \mathrm{~s}^{-1}$, explaining the concentration of the BGK00 distribution toward greater $H$ luminosities. Very low luminosity objects appear predominantly in the SSM04 sample because BGK00 have a lower redshift cutoff of $2300 \mathrm{~km} \mathrm{~s}^{-1}$, which eliminates these objects. We take $H_{0}=$ $73 \mathrm{~km} \mathrm{~s}^{-1} \mathrm{Mpc}^{-1}$ for all luminosity comparisons.

Figure 16 shows that the range of $\log L_{\mathrm{FIR}}$ in the SSM04 sample is 9.1-11.8; in the BGK00 sample, the range is 9.3-11.4. As in Figure 15, the core of the SSM04 luminosity distribution is broader. The $\left\langle\log L_{\mathrm{FIR}}\right\rangle$ in the SSM04 sample is 10.5, whereas in the BGK00 sample $\left\langle\log L_{\mathrm{FIR}}\right\rangle$ is 10.2 . The shift toward a broader distribution with a greater $\left\langle\log L_{\mathrm{FIR}}\right\rangle$ in the SSM04 sample is as expected for an $I R A S$-selected sample relative to a $B$-selected sample. Neither sample contains ULIRGs.

Figure 17 shows the distributions of $\log \left(L_{\mathrm{FIR}} / L_{H}\right)$, a measure of the normalized star formation rate for both the SSM04 and BGK00 samples. The $L_{\mathrm{FIR}}$ value is directly proportional to the star formation rate (Kennicutt 1998; Calzetti et al. 2000; Charlot et al. 2002; Kewley et al. 2002), and $L_{H}$ is roughly proportional to the stellar mass. Some investigators use $L_{K}$ to normalize star 

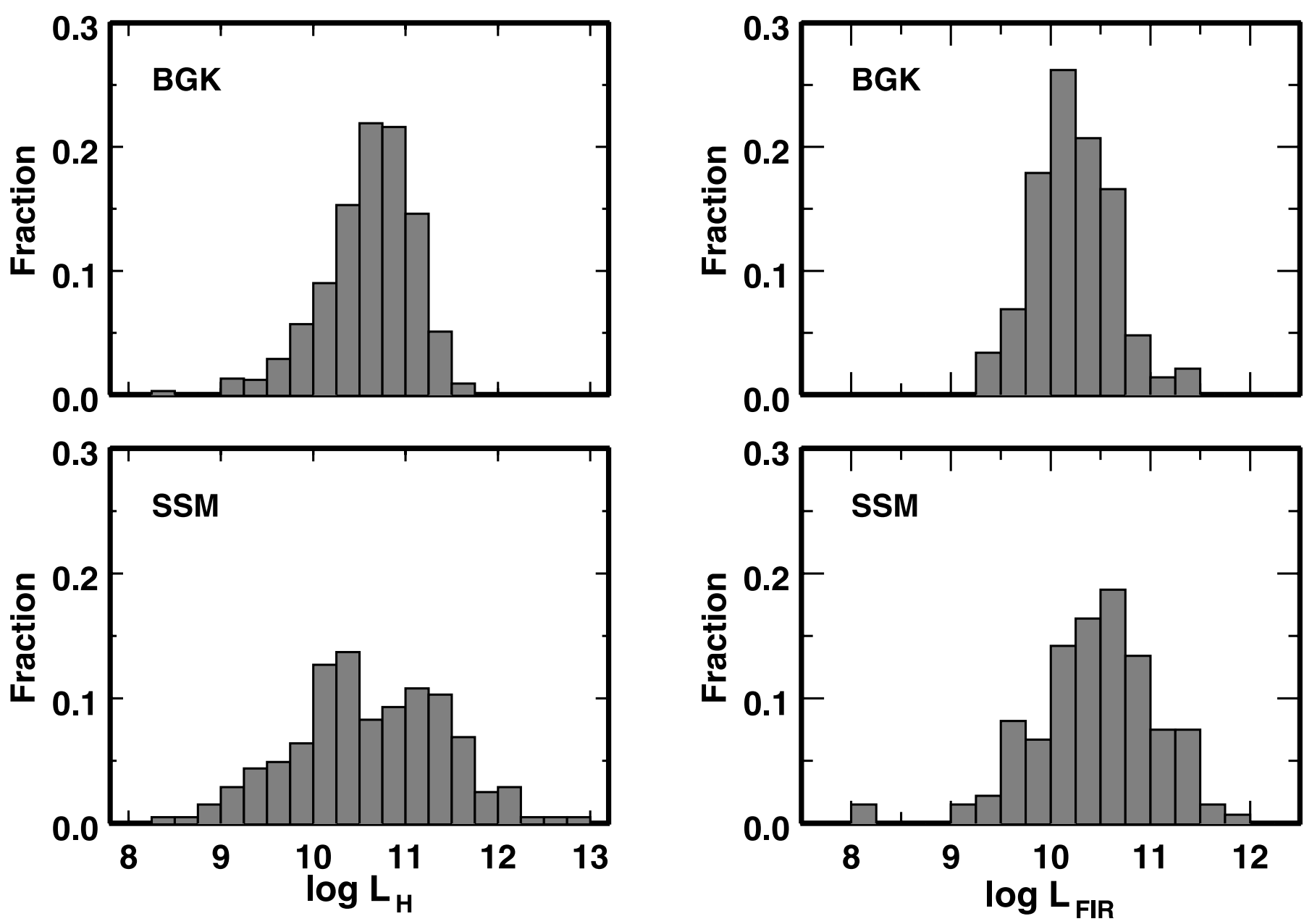

FIG. 15.-Normalized distributions of $L_{H}$ for individual galaxies in the BGK00 and SSM04 pair samples. Note the broader distribution of SSM04 luminosities.

FIG. 16.- Normalized distributions of $L_{\mathrm{FIR}}$ for BGK00 and SSM04 pairs. Note the extension of the SSM04 luminosity distribution into the ULIRG range. 

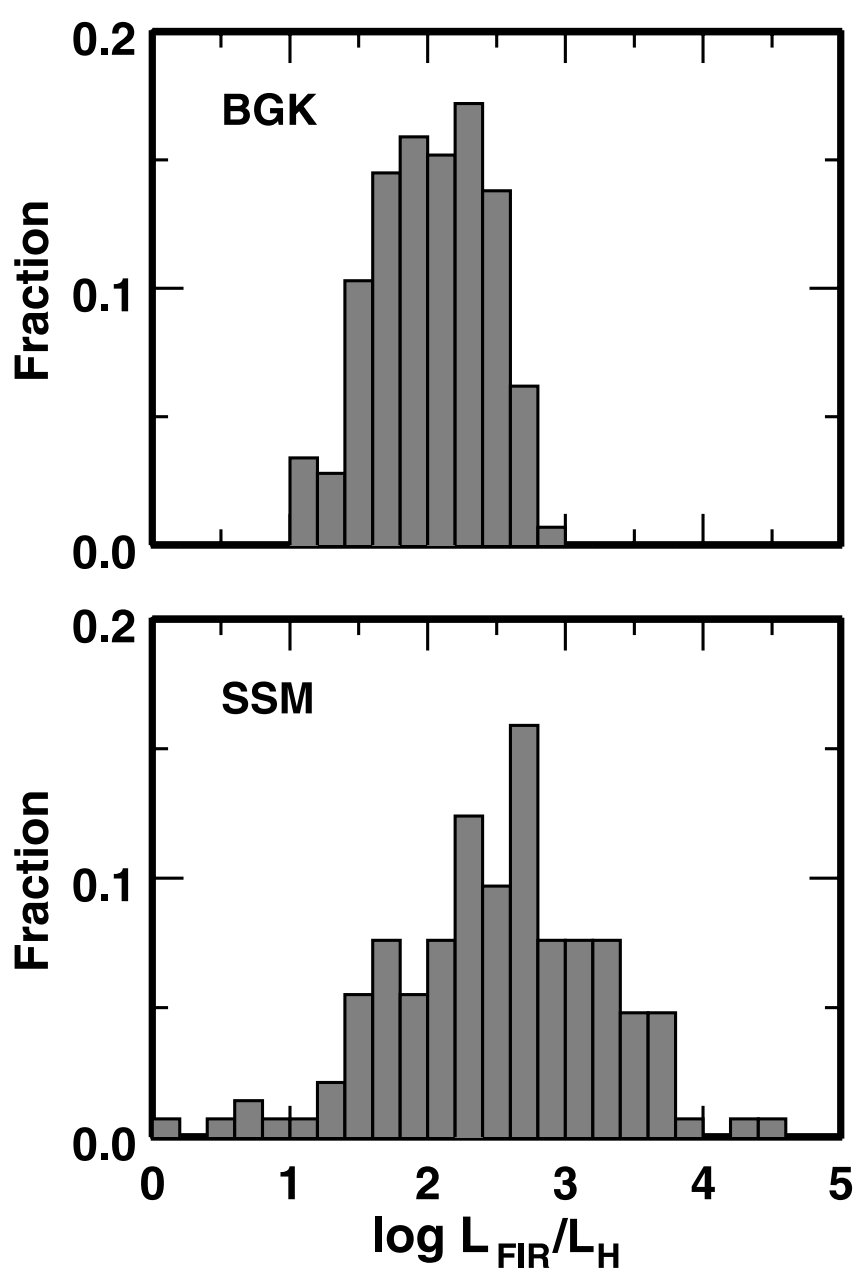

Fig. 17. - Normalized distributions of $L_{\mathrm{FIR}} / L_{H}$ for the BGK00 and SSM04 pairs.

formation rates. As we have discussed, $L_{K}$ is affected by thermal emission from hot dust in these systems, and thus, $L_{H}$ is a preferable proxy for the stellar mass.

In Figure 17, $L_{H}$ is the $H$-band luminosity for the pair derived from 2MASS photometry. Other investigators have shown that the IRAS luminosities of close pairs are frequently dominated by one of the galaxies. In the BGK00 sample, nearly all of the pairs are unresolved in $I R A S$, and we have no way of knowing the detailed origin of the IRAS emission. We thus normalize the $I R A S$ luminosity by the total $H$-band luminosity for the pair. This approach may systematically underestimate the normalized star formation rate, but because most pairs are galaxies of comparable $H$-band luminosity, the bias is on the order of a factor of 2 .
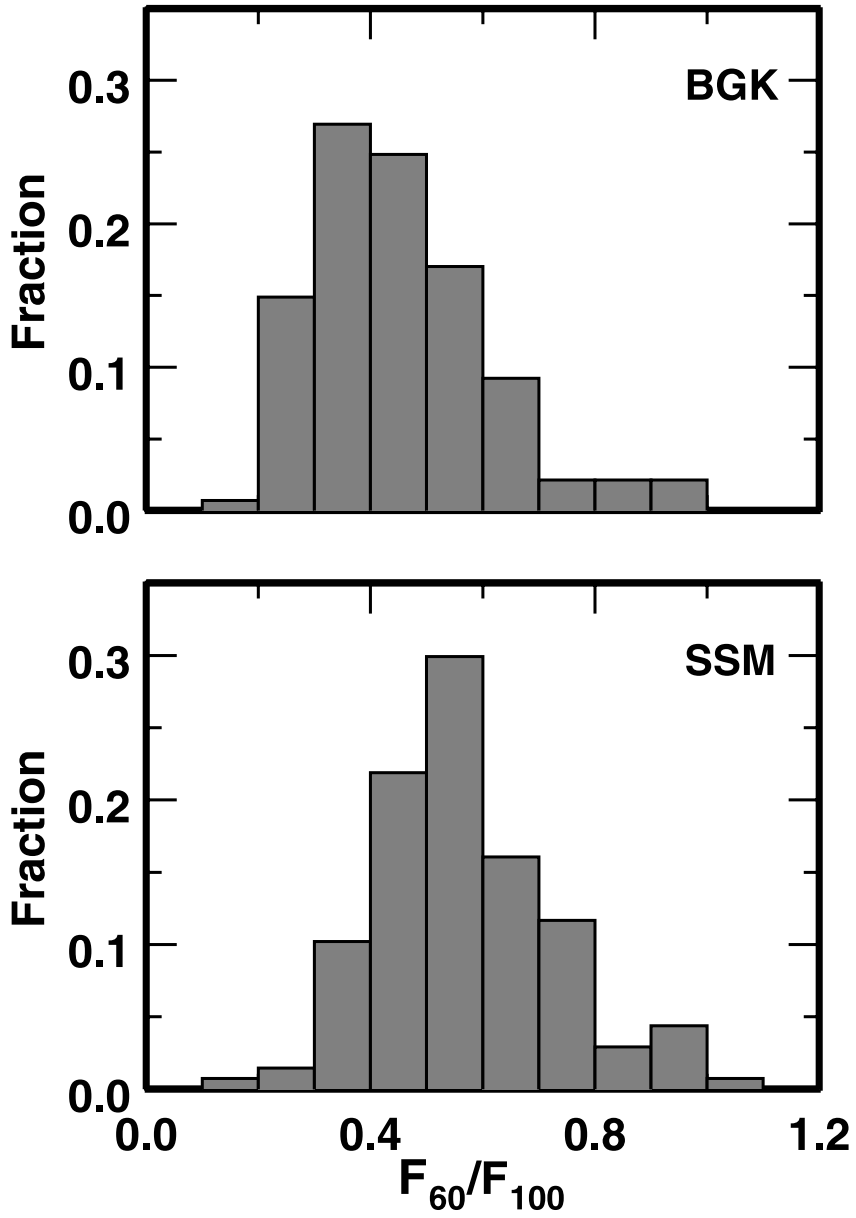

FIG. 18.-Normalized distributions of $F_{60} / F_{100}$ for the BGK00 and SSM04 pairs.

The difference in the distributions is more pronounced in Figure 17 than in Figure 16. The IRAS selection yields a sample rich in pairs with $\log \left(L_{\mathrm{FIR}} / L_{H}\right) \geq 2.8$. These objects are too faint at $B$ to be included in the BGK00 sample. The tail at $\log \left(L_{\mathrm{FIR}} / L_{H}\right) \leq 1$ in the SSM04 sample consists of low-redshift objects absent by construction from the BGK00 sample.

The SSM04 IRAS-selected pairs also contain somewhat hotter dust than the BGK00 pairs detected by IRAS. Figure 18 shows the distributions of $F_{60} / F_{100}$ for both samples. The median temperature of the BGK00 pairs is $45 \mathrm{~K}$; the median for the SSM04 pairs is $50 \mathrm{~K}$.

In general, the BGK00 sample contains less extreme objects than the SSM04 sample, but the overlap is substantial over 2 orders of magnitude in both $L_{\mathrm{FIR}}$ and $L_{\mathrm{FIR}} / L_{H}$. Not surprisingly, pairs with $\log \left(L_{\mathrm{FIR}} / L_{H}\right) \geq 2.8$ are rare in a $B$-selected sample like BGK00.
Aaronson, M. 1977, Ph.D. thesis, Harvard Univ.

Allam, S. S., Tucker, D. L., Smith, J. A., Lee, B. C., Annis, J., Lin, H., Karachentsev, I. D., \& Laubscher, B. E. 2004, AJ, 127, 1883

Alonso, M. S., Tissera, P. B., Coldwell, G., \& Lambas, D. G. 2004, MNRAS, 352, 1081

Alonso-Herrero, A., et al. 2006, ApJ, 640, 167

Arp, H. C., \& Madore, B. 1987, A Catalogue of Southern Peculiar Galaxies and Associations (Cambridge: Cambridge Univ. Press)

Balog, Z., Kenyon, S. J., Lada, E. A., Barsony, M., Vinkó, J., \& Gáspaŕ, A. 2004, AJ, 128, 2942

Barsony, M., Kenyon, S. J., Lada, E. A., \& Teuben, P. J. 1997, ApJS, 112, 109

Barton, E. J., Geller, M. J., \& Kenyon, S. J. 2000, ApJ, 530, 660 (BGK00)

\section{REFERENCES}

Barton Gillespie, E., Geller, M. J., \& Kenyon, S. J. 2003, ApJ, 582, 668 (BGK03)

Beichman, C., et al., eds. 1985, IRAS Catalogs and Atlases (Washington: USGPO)

Bekki, K., \& Couch, W. J. 2001, ApJ, 557, L19

Bell, E. F., et al. 2006, ApJ, 640, 241

Bessell, M. S., \& Brett, J. M. 1988, PASP, 100, 1134

Bragg, A. E., \& Kenyon, S. J. 2002, AJ, 124, 3289

Bushouse, H. A., \& Werner, M. W. 1990, ApJ, 359, 72

Bushouse, H. A., Werner, M. W., \& Lamb, S. A. 1988, ApJ, 335, 74

Calzetti, D., Armus, L., Bohlin, R. C., Kinney, A. L., Koornneef, J., \& StorchiBergmann, T. 2000, ApJ, 533, 682 
Campbell, A. W., \& Terlevich, R. 1984, MNRAS, 211, 15

Carico, D. P., Sanders, D. B., Soifer, B. T., Matthews, K. \& Neugebauer, G. 1990, AJ, 100, 70

Carico, D. P., Soifer, B. T., Beichman, C., Elias, J. H., Matthews, K., \& Neugebauer, G. 1986, AJ, 92, 1254

Charlot, S., Kauffmann, G., Longhetti, M., Tresse, L., White, S. D. M., Maddox, S. J., \& Fall, S. M. 2002, MNRAS, 330, 876

Conselice, C. J., et al. 2004, ApJ, 600, L139

Corbett, E. A., et al. 2003, ApJ, 583, 670

Cutri, R. M., \& McAlary, C. W. 1985, ApJ, 296, 90

Devereux, N. A. 1989, ApJ, 346, 126

Donzelli, C. J., \& Pastoriza, M. G. 1997, ApJS, 111, 181

Elias, J. H., Frogel, J. A., Matthews, K., \& Neugebauer, G. 1982, AJ, 87, 1029

Falco, E. E., et al. 1999, PASP, 111, 438

Fruchter, A. S., \& Hook, R. N. 2002, PASP, 114, 144

Gallagher, J. S., \& Smith, L. J. 1999, MNRAS, 304, 540

Geller, M. J., \& Huchra, J. P. 1989, Science, 246, 897

Giovanelli, R., \& Haynes, M. P. 1985, AJ, 90, 2445

1989, AJ, 97, 633

1993, AJ, 105, 1271

Giovanelli, R., Myers, S. T., Roth, J., \& Haynes, M. P. 1986, AJ, 92, 250

Giuricin, G., Biviano, A., Girardi, M., Mardirossian, F., \& Mezzetti, M. 1993, A\&A, 275, 390

Glass, I. S., \& Moorwood, A. F. M. 1985, MNRAS, 214, 429

Gómez, M., \& Kenyon, S. J. 2001, AJ, 121, 974

Goto, T. 2005, MNRAS, 360, 322

Griersmith, D., Hyland, A. R., \& Jones, T. J. 1982, AJ, 87, 1106

Guiderdoni, B., Hivon, E., Bouchet, F. R., \& Maffei, B. 1998, MNRAS, 295, 877

Haynes, M. P., Magri, C., Giovanelli, R., \& Starosta, B. M. 1988, AJ, 95, 607

Helou, G., Khan, I. R., Malek, L., \& Boehmer, L. 1988, ApJS, 68, 151

Helou, G., Lu, N. Y., Werner, M. W., Malhotra, S., \& Silbermann, N. 2000, ApJ, 532, L21

Huchra, J. P., Geller, M. J., \& Corwin, H. G. 1995, ApJS, 99, 391

Huchra, J. P., Geller, M. J., de Lapparent, V., \& Corwin, H. G. 1990, ApJS, 72, 433

Huchra, J. P., Vogeley, M. S., \& Geller, M. J. 1999, ApJS, 121, 287

Hunt, L. K., Giovanardi, C., \& Helou, G. 2002, A\&A, 394, 873

Jansen, R. A., Fabricant, D., Franx, M., \& Caldwell, N. 2000a, ApJS, 126, 331

Jansen, R. A., Franx, M., Fabricant, D., \& Caldwell, N. 2000b, ApJS, 126, 271

Jarrett, T. H. 2000, PASP, 112, 1008

Jarrett, T. H., Chester, T., Cutri, R., Schneider, S., Skrutskie, M., \& Huchra, J. P. 2000, AJ, 119, 2498

Jones, B., \& Stein, W. A. 1989, AJ, 98, 1557

Kassin, S. A., Frogel, J. A., Pogge, R. W., Tiede, G. P., \& Sellgren, K. 2003, AJ, 126, 1276

Keel, W. C. 1993, Rev. Mex. AA, 27, 77 1996, ApJS, 106, 27

Keel, W. C., \& Borne, K. D. 2003, AJ, 126, 1257
Kennicutt, R. C., Jr. 1998, ARA\&A, 36, 189

Kennicutt, R. C., Jr., \& Keel, W. C. 1984, ApJ, 279, L5

Kennicutt, R. C., Jr., Roettiger, K. A., Keel, W. C., van der Hulst, J. M., \& Hummel, E. 1987, AJ, 93, 1011

Kewley, L. J., Dopita, M. A., Sutherland, R. S., Heisler, C. A., \& Trevena, J. 2001a, ApJ, 556, 121

Kewley, L. J., Geller, M. J., \& Barton, E. J. 2006, AJ, 131, 2004

Kewley, L. J., Geller, M. J., Jansen, R. A., \& Dopita, M. A. 2002, AJ, 124, 3135

Kewley, L. J., Heisler, C. A., Dopita, M. A., \& Lumsden, S. 2001b, ApJS, 132, 37

Kewley, L. J., Jansen, R. A., \& Geller, M. J. 2005, PASP, 117, 227

Lambas, D. G., Tissera, P. B., Alonso, M. S., \& Coldwell, G. 2003, MNRAS, 346, 1189

Larson, R. B., \& Tinsley, B. M. 1978, ApJ, 219, 46

Lavalley, M., Isobe, T., \& Feigelson, E. 1992, in ASP Conf. Ser. 25, Astronomical Data Analysis Software and Systems I, ed. D. M. Worrall, C. Biemesderfer, \& J. Barnes (San Francisco: ASP), 245

Lin, L., et al. 2004, ApJ, 617, L9

Liu, C. T., \& Kennicutt, R. C. 1995a, ApJS, 100, 325 1995b, ApJ, 450, 547

López-Sánchez, Á. R., Esteban, C., \& Rodríguez, M. 2004, ApJS, 153, 243

Marzke, R. O., Geller, M. J., da Costa, L. N., \& Huchra, J. P. 1995, AJ, 110, 477

Mihos, J. C., \& Hernquist, L. 1996, ApJ, 464, 641

Mihos, J. C., Richstone, D. O., \& Bothun, G. D. 1991, ApJ, 377, 72

Moorwood, A. F. M., Veron-Cetty, M.-P., \& Glass, I. S. 1987, A\&A, 184, 63

Nikolaev, S., Weinberg, M. D., Skrutskie, M. F., Cutri, R. M., Wheelock, S. L., Gizis, J. E., \& Howard, E. M. 2000, AJ, 120, 3340

Nikolic, B., Cullen, H., \& Alexander, P. 2004, MNRAS, 355, 874

Papovich, C., Dickinson, M., Giavalisco, M., Conselice, C. J., \& Ferguson, H. C. 2005, ApJ, 631, 101

Patton, D. R., et al. 2002, ApJ, 565, 208

Poggianti, B. M. 1997, A\&AS, 122, 399

Press, W. H., Teukolsky, S. A., Vetterling, W. T., \& Flannery, B. P. 1992, Numerical Recipes in C: The Art of Scientific Computing (2nd ed.; Cambridge: Cambridge Univ. Press)

Ramos-Larios, G., \& Phillips, J. P. 2005, MNRAS, 357, 732

Sanders, D. B., \& Mirabel, I. F. 1996, ARA\&A, 34, 749

Sekiguchi, K., \& Wolstencroft, R. D. 1992, MNRAS, 255, 581

Skrutskie, M. F., et al. 2006, AJ, 131, 1163

Surace, J. A., Sanders, D. B., \& Mazzarella, J. M. 2004, AJ, 127, 3235 (SSM04)

Telesco, C. M., Wolstencroft, R. D., \& Done, C. 1988, ApJ, 329, 174

Veilleux, S., Kim, D.-C., Sanders, D. B., Mazzarella, J. M., \& Soifer, B. T. 1995, ApJS, 98, 171

Wegner, G., Haynes, M. P., \& Giovanelli, R. 1993, AJ, 105, 1251

Whitelock, P. A. 1985, MNRAS, 213, 59

Whitney, B. A., Kenyon, S. J., \& Gomez, M. 1997, ApJ, 485, 703

Zwicky, F., et al. 1968, Catalog of Galaxies and of Clusters of Galaxies (Pasadena: Caltech) 